\title{
Control of Quantum Dynamics: Concepts, Procedures and Future Prospects
}

\author{
Herschel Rabitz \\ Department of Chemistry, Princeton University, Princeton, NJ 08544-1009, USA \\ Gabriel Turinici \\ INRIA Rocquencourt, B.P. 105, 78153 Le Chesnay Cedex France \\ and CERMICS-ENPC, Champs sur Marne, 77455 Marne la Vallée Cedex, France \\ Eric Brown \\ Program in Applied and Computational Mathematics, 205 Fine Hall,Princeton \\ University, Princeton, NJ 08544-1009, USA
}

Key words: quantum control theory, quantum dynamics, inverse problems

\section{Contents}

Chapter 1. Introduction 3

$1 \quad$ Historical perspective on quantum control 3

2 Multidisciplinarity 3

3 Outline 4

$\begin{array}{ll}\text { Chapter 2. Basic principles } & 4\end{array}$

4 Mathematical formulation of the Hamiltonian and control law 4

$\begin{array}{lll}5 & \text { Optimal control } & 7\end{array}$

$\begin{array}{lll}6 & \text { More nonlinear formulations } & 7\end{array}$

Email addresses: hrabitz@princeton.edu (Herschel Rabitz), Gabriel.Turinici@inria.fr (Gabriel Turinici), ebrown@princeton.edu (Eric Brown).

URLs: http://www.princeton.edu/ hrabitz (Herschel Rabitz), http://www-rocq.inria.fr/Gabriel.Turinici (Gabriel Turinici).

$\begin{array}{ll}\text { Preprint submitted to Elsevier Science } & 3 \text { July } 2002\end{array}$ 
$\begin{array}{llr}7 & \text { Density matrix formulation } & 8\end{array}$

8 Special control schemes: pump-dump schemes, STIRAP 9

$9 \quad$ Classical mechanics formulations $\quad 9$

$\begin{array}{ll}\text { Chapter 3. Controllability of Quantum Mechanical Systems } & 10\end{array}$

10 Infinite dimensional controllability $\quad 12$

11 Finite dimensional controllability $\quad 12$

12 Truncations 23

$\begin{array}{ll}\text { Chapter 4. Quantum control algorithms } & 25\end{array}$

13 Optimal control cost functional formulation 26

14 Numerical algorithms $\quad 29$

15 Robust designs 36

$\begin{array}{lll}16 & \text { Tracking } & 39\end{array}$

17 Laboratory achievement of closed loop control 40

Chapter 5. Challenges for the future 45

18 Coherence and control 45

19 Can noise help attain control ? 46

20 Qualitative behavior of optimal control solutions 46

$\begin{array}{lll}21 & \text { Feedback quantum control } & 47\end{array}$

22 Algorithms for the inversion of quantum dynamics data $\quad 50$

23 Identification of quantum control mechanism and "rules of thumb" 52

$\begin{array}{ll}\text { Chapter 6. Conclusion } & 53\end{array}$ 


\section{Chapter 1. Introduction}

\section{Historical perspective on quantum control}

Along with the development of laser technology, a rising interest has naturally appeared in connection with using lasers to influence matter at the quantum dynamical level. This new field of research, commonly designated as "quantum control", has roots that go back to the earliest days of laser development in the 1960's. What distinguishes quantum control from the traditional means of chemical manipulation is the use of delicate quantum wave interferences to alter the outcome of molecular scale dynamics phenomena. Research in quantum control accelerated in the 1980's, with the first successful results in Shi et al. (1988); Tannor and Rice (1985); Shapiro and Brumer (1989) driven by the recognition of the role of quantum interference and the need for rigorous design tools. The introduction of the evolutionary paradigm (see Section 17) in Judson and Rabitz (1992) and the accompanying experimental successes of Assion et al. (1998); Levis et al. (2001); Weinacht et al. (1999); Bardeen et al. (1997a, 1998); Hornung et al. (2000); Kunde et al. (2000) in the 1990's have brought an exciting new set of theoretical questions and laboratory possibilities to the field. The time scales involved lie mostly within the femtosecond $\left(10^{-15}\right)$ - picosecond $\left(10^{-12}\right)$ range with spatial dimensions extending from one or two atoms to large polyatomic molecules and solid state structures.

In practice the outcome of a control experiment is measured in terms of quantum observables (e.g. selective dissociation of interatomic bonds) associated with Hermitian operators acting on the the system at hand. The control proocess consists of steering the appropriate observable or wavefunction from the initial state to a final desired state. In the laboratory this is generally done by time-varying laser fields. We refer the reader to Chapter 2 for a precise mathematical transcription of these concepts.

Although prospects for industrial applications motivated much of the early research on quantum control, current applications span a wide range, from high harmonic generation (Bartels et al. (2000)) and fast switching in semiconductors (Kunde et al. (2000)) to Hamiltonian identification (see Section 22).

\section{Multidisciplinarity}

Although research on quantum control was initiated within the field of physical chemistry, the subject has developed to involve researchers from myriad fields, including engineering, mathematics, computer science and physics. The 
introduction of control tools from the engineering contexts opened the field to the influence of (applied) mathematicians; later, computer scientists also became involved through their interest in quantum computing. Besides techniques employed by control specialists working on controllability, observability and stabilization issues, the applied mathematics tools involved include the resolution of the control problem through deterministic (direct optimization or critical point equations) or stochastic (evolutionary algorithms) approaches. The variety of research paradigms contributing to quantum control has proved to be beneficial to the field and will likely be central to future advances.

\section{Outline}

This volume addresses numerical methods in various aspects of the chemical sciences and the present chapter contributes in that fashion to the subject of quantum control. However, in order to appreciate how numerical methods are relevant for quantum control it is essential to understand how they fit into the overall subject including its laboratory implementations. Thus, the chapter attempts to give a full perspective on the subject including its theoretical foundations as well as its current state in the laboratory.

The balance of this review will proceed as follows: after an introduction of the fundamental concepts in Chapter 2 we present in Chapter 3 theoretical results regarding on the controllability of bilinear quantum systems. Then, in Chapter 4 we present the available numerical and experimental algorithmical approaches employed to control quantum phenomena. Present topics of interest and open questions are the object of the Chapter 5. Concluding remarks are presented in Chapter 6. The text draws on many works in the literature and especially a recent prospectus (Brown and Rabitz (in press)) on the field.

\section{Chapter 2. Basic principles}

\section{Mathematical formulation of the Hamiltonian and control law}

Each particular control setting requires an appropriate quantum control model. The section will be mostly devoted to the description of the bilinear dipole coupling, which is often employed in practice. However, some other control paradigms will be presented and their domain of applicability and specific capabilities discussed. Controlling a quantum system requires the introduction of external interactions. Here, we will only consider control tools that act at 
the atomic/molecular level, with other, more classical tools such as temperature, pressure, catalysts etc. being outside of our scope. The primary control for quantum systems discussed here will be the electric field of a laser.

Most of this paper will work under the assumption that the system to be controlled can be characterized by its state function $\psi(t)$. This is a proper representation for isolated systems starting in a pure state; the complementary case arises, for example, in collisional or condensed regimes, when a density operator $\rho(t)$ must be introduced to describe the statistical mixture of states making up the system. The density operator formulation will be discussed where relevant.

Consider a quantum system that evolves from the initial state $\psi(t=0)=\psi_{0}$. The objective of quantum control can generally be expressed as the desire for the expectation value

$$
\langle O(t)\rangle=\langle\psi(t)|O| \psi(t)\rangle
$$

of some predefined observable operator $O$ to be within a prescribed target set at the final time $t=T$. More general quantum control problem formulations may deal with several observable operators $O_{1}, \ldots, O_{k}(k>1)$ and additional intermediary times $0 \leq t_{j} \leq T(j=1,2, \ldots)$.

In the absence of any external control influence, evolution of the state function $\psi(t)$ under the Schrödinger equation is determined by the free Hamiltonian $H_{0}$, which by assumption does not yield dynamics producing the desired expectation values. Quantum control theory considers the addition of a laboratory accessible control law term $C(t)$ to the Hamiltonian in order to achieve these objectives, which makes $H=H_{0}+C(t)$ the new Hamiltonian of the system and

$$
i \hbar \frac{\partial \psi(t)}{\partial t}=\left[H_{0}+C(t)\right] \psi(t)
$$

the equation of motion. Appropriate regularity assumptions on the control law must be enforced so that the evolution of $\psi(t)$ is well defined. For example, a common control law for lasers has the form

$$
C(t)=-\mu \epsilon(t), \epsilon=\left(\epsilon_{i}\right)_{i=1}^{3}, \epsilon_{i}(t) \in L^{2}[0, T], i=1,2,3
$$

where $\mu$ is the electric dipole operator, $\epsilon(t)$ is the applied electric field, and the index $i$ refers to spatial orientation.

Remark 1 Depending on the problem, one may go beyond the first-order, bilinear term in Eqn. (4.2) when describing the interaction between the laser and the system, cf. Dion et al. (1999a,b).

Additional admissibility conditions may ensure that $\epsilon(t)$ obeys laboratory limitations on the range of achievable laser frequencies, intensities, energy, or other 
criteria. In some applications, additional possibilities for $C(t)$ arise. These include

- the use of magnetic fields, in which case the control law becomes $-\mu_{m} B(t)$, where $\mu_{m}$ is the magnetic dipole operator and $B(t)$ is the magnetic field, and

- the use of materials whose design specifications themselves take the form of a control law, such as for quantum electron transport in semiconductors with variable material composition considered as the control.

Here, however, we will confine the discussion to time-dependent controls based on an external electric field $\epsilon(t)$ coupled to the system through a dipole $\mu$.

In some cases, it may be possible to obtain an adequate control description by replacing the Schrödinger equation with a classical representation of the system dynamics (see Section 9). This is especially true for interatomic phenomena, because the de Broglie wavelength associated with atoms is often short relative to interatomic length scales. While the relationships between classical and quantum models of molecular evolution have been extensively investigated, e.g. Davis and Heller (1984); Kulander and Orel (1981); Leforestier (1986); Dardi and Gray (1982), the implications of these relationships for control are not completely understood and will be addressed later in the context of the quantum character of the control problem.

Assuming knowledge of $H_{0}$ and a well-defined control law $C(t)$, Eqn. (4.2) or its classical equivalent represents a complete model of the system of interest. If $C(t)$ is given a priori, the solution of Eqn. (4.2) is a standard numerical problem in time-dependent quantum mechanics. However, the essence of the control problem is to find $C(t)$ such that the objectives in Eqn. (4.1) are met, and since at least one of the control objectives lies in the future, this task presents some additional challenges. In particular, the Hamiltonian depends on the future state of the system through the control objectives, as can be formally represented by the expression $C(t)=C(\psi(s): s \in[t, T])$. This non-causality introduces an entirely new set of mathematical issues which are not present in standard quantum or classical dynamics but are inherent to the theory and practice of temporal control in engineering and mathematical systems theory (cf. Sontag (1998); Brockett (1970); Kailath (1980); Khalil (1996); Nijmeiher and van der Schaft (1990)). Their implications for the quantum regime are central issues. The formulation above is summarized in the following definition:

Definition 2 The quantum control problem consists of determining a control law $C(t)$ that causes the system to optimally achieve the desired expectation values while possibly also satisfying auxiliary conditions. Quantum control theory encompasses methods of determining these control laws, their general properties, and their relationship to the underlying physical system and evolving 


\section{Optimal control}

Several approaches for determining control laws $C(t)$ will be discussed in this chapter. The present section introduces the concept of optimal control theory for this purpose; a more detailed algorithmic analysis will be given in Chapter 4. An extensive literature on optimal control theory can be found in classical engineering and mathematical systems theory (e.g., Sontag (1998); Luenberger (1979); Mohler (1983)) and increasingly in quantum mechanics (e.g. Peirce et al. (1988); Zhao and Rice (1994), Ch. 6 of Rice and Zhao (2000) and references therein). Considering control law design as an optimization problem is quite natural, as attaining the best possible final level of control is always the goal; further, optimization is essential when there are competing physical objectives that must simultaneously be met.

The optimal control approach seeks to optimize a cost functional $J$, which includes both terms that describe how well the objective has been met and terms that penalize undesirable effects. One simple example of a cost functional is

$$
J(\epsilon)=<\psi(T)|O| \psi(T)>-\alpha \int_{0}^{T} \epsilon^{2}(t) d t
$$

where $\alpha>0$ is a parameter and $O$ is the observable operator (positive semidefinite in this case) that specifies the goal. In mathematical terms, the observable $O$ is a self-adjoint operator that acts on $\psi(T)$; in the case above, the goal is to achieve a large the value $\langle\psi(T)|O| \psi(T)>$. Note that in general attaining the maximum possible value of $\langle\psi(T)|O| \psi(T)>$ comes at the price of a large laser fluence $\int_{0}^{T} \epsilon^{2}(t) d t$, so that the optimum evolution will strike a balance (weighted by $\alpha$ ) between laser fluence and operator expectation values.

\section{More nonlinear formulations}

The quantum control problem becomes more complex when the control law and the free Hamiltonian cannot be treated independently. An important example of this phenomenon is intense-field laser control of molecular motion, where the electric field can directly alter the dipole operator through its manipulation of the electronic degrees of freedom:

$$
C(t)=-\mu(\epsilon(t)) \epsilon(t) .
$$


In simple cases, the relation in Eqn. (6.1) may be expanded in terms of a low order polynomial in $\epsilon(t)$ whose coefficients are the electric moments and polarizabilities of the system. Of special interest are situations in which the nonlinear structure may affect the controllability of the system (including the positive case in which this interaction makes a previously inaccessible target reachable).

Remark 3 To date, very few mathematical studies exist to treat the situation where the control law has a nonlinear dependence on the control field; it is not clear whether successful approaches will draw upon existing methodology for proving, for example, quantum controllability (see Chapter 3) or upon new tools from mathematical systems theory.

The circumstances motivating Remark 3 can also be viewed from the larger perspective of a controllability analysis simultaneously including electronic and nuclear motion e.g. as in Cancès and Le Bris (1999). In the latter circumstance the control field will enter the Hamiltonian linearly, but at the expense of explicitly including the electronic degrees of freedom. The same comment also applies to the performance of optimal control designs in the strong-field regime. The practical importance of investigating the latter domain has recently been demonstrated experimentally (Levis et al. (2001)).

A different formulation is necessary when the back action of the quantum medium upon the propagating control field is significant. In this case the medium is called optically dense. This scenario has been examined experimentally for a vapor of sodium (Shen et al. (1993)), and the topic is of practical importance because the controlled medium will be dense in any application directed toward collecting large amounts of product. Optically dense media can interact with the electric field to alter its phase and/or amplitude structure as it propagates. In order to model this effect, the Schrödinger equation must be coupled with Maxwell's equations e.g. Wang and Rabitz (1996).

\section{Density matrix formulation}

In practical applications the medium will be at a finite temperature; in this case (and in any situation that concerns a statistical mixture of quantum states) the density operator formulation is necessary. In this formulation, the time evolution is given by the quantum Liouville equation (Cohen-Tannoudji et al. (1997)):

$$
\frac{d \rho(t)}{d t}=\frac{1}{i \hbar}[H(t), \rho(t)]
$$

and expectation values are calculated as

$$
\langle O(t)\rangle=\operatorname{Tr}(\rho(\mathrm{t}) \mathrm{O}) .
$$


Upon introducing a cost functional as in Eqn. (5.1), quantum systems described by density operators can also be treated by optimal control theory.

\section{Special control schemes: pump-dump schemes, STIRAP}

In addition to the general approach outlined below, special techniques for control law design have been developed for cases in which a priori specification of control mechanisms is possible. These methods include the weak-field regime, time-resolved "pump-dump" and simulated Raman adiabatic passage (STIRAP) schemes. Under particular quantum dynamics approximations and/or assumptions, these techniques allow for the derivation of closed form expressions for control laws that optimally accomplish certain control objectives under their specified conditions. The literature on related theoretical developments is extensive and will not be presented here (Ch. 3-5 of Rice and Zhao (2000) and references therein, Brown and Sibley. (1998); Shah and Rice (1999); Mishima and Yamashita. (1999b); Hoki et al. (1999); de Araujo and Walmsley (1999); Mishima and Yamashita. (1999a); Cao and Wilson. (1997); Bardeen et al. (1997c,b); Agarwal (1997); Gronager and Henriksen. (1998)) and experimental (Ch. 3-5 of Rice and Zhao (2000) and references therein, Bardeen et al. (1997c,b); Meshulach and Silberberg. (1998); Pastirk et al. (1998)).

\section{Classical mechanics formulations}

Classical modeling of quantum systems is a common and often successful technique, and it should have a level of applicability in molecular control. This section attempts to discuss its applicability, or the quantum character of molecular scale control. Nonclassical characteristics of dynamical behavior include tunneling, quantization of energy levels, and interference processes, but it is not clear which of these characterizations are most relevant to defining the quantum nature of a control problem. Understanding of this issue could be used to estimate the loss of reliability (i.e., defined upon comparison to the analogous quantum system response to the classically designed field) in resorting to the classical optimal control formulation.

Some aspects of this topic have been addressed in Schwieters and Rabitz (1993), where quantum $C_{q}(t)$ and classical $C_{c}(t)$ control laws corresponding to equivalent representations of specific control problems are compared. The equations of motion analogous to Eqn. (4.2) are:

$$
\frac{d q_{i}^{l}}{d t}=\frac{\partial H}{\partial p_{i}^{l}}, \quad \frac{d p_{i}^{l}}{d t}=-\frac{\partial H}{\partial q_{i}^{l}},
$$


and expectation values for the classical system are given by

$$
\left\langle O_{c}\right\rangle=\sum_{l=1}^{N_{c}} \Gamma_{l} \bar{O}\left(\mathbf{q}^{l}, \mathbf{p}^{l}\right),
$$

where $H$ is the classical Hamiltonian of the system, $i$ ranges over the particle coordinates, $l$ indexes initial conditions $q^{l}(0), p^{l}(0)$, and the weights $\Gamma_{l}$ for the $N_{c}$ initial conditions are chosen to mimic as best as possible the probability distribution function for the corresponding quantum system. Here, $\bar{O}$ is a classical observable corresponding to its quantum analog. It should be noted that the ordinary differential equations in Eqn. (9.1) for some cases may be more expensive to solve than their quantum counterpart in Eqn. (4.2). One motivation for considering classical optimal control design is for the physical insight possible with classical mechanics.

Optimal control theory has been used e.g. in Schwieters and Rabitz (1993) to separately design a control field $\epsilon(t)$ that minimizes the difference between $\langle O\rangle$ and $\left\langle O_{c}\right\rangle$ and the difference between each of these expectation values and the control objectives on $[0, T]$. For the example of a Morse oscillator, it was found that an optimal control law designed in this fashion produced very close agreement between $\langle O\rangle$ and $\left\langle O_{c}\right\rangle$. This result suggests that in some cases classically-designed controls can also be successful as quantum controls. In related work Schwieters and Rabitz (unpublished results), a method was developed for determining potentials under which evolving classical and corresponding quantum systems give similar values of classical and quantum observables; the approach met with considerable success for the control of dissociative flux and displacement. In general it is however not known for what classes of Hamiltonians and control objectives the quantum control problem can be adequately addressed using classical equations of motion. Because interference itself is a nonclassical phenomenon, this problem is related to the considerations of decoherence in the Section 18.

\section{Chapter 3. Controllability of Quantum Mechanical Systems}

Prior to addressing the computation of the control law, it is natural to ask if the quantum control problem is well-posed such that a control law exists which will cause the objectives and auxiliary conditions to be (precisely) satisfied. Even if the answer to the latter question is negative, one may still be satisfied with achieving the control objectives (maybe only partially) through the techniques of optimal control. The fundamental importance of addressing controllability has long been recognized in engineering control applications; the broad literature on the classical aspects of the subject includes many comprehensive texts which cover linear (Brockett (1970); Kailath (1980)) and nonlinear (Sontag (1998); Khalil (1996); Nijmeiher and van der Schaft (1990)) 
controllability. In addition, several works have considered various aspects of quantum controllability (e.g., Rice and Zhao (2000); Huang et al. (1983); Judson et al. (1990); Ramakrishna et al. (1995); Turinici (2000c); Turinici and Rabitz (2001a,b); Girardeau et al. (1998, 1997)).

Consider a quantum system (isolated from external influences for the moment) with internal Hamiltonian $H_{0}$ that is prepared in the initial state $\psi_{0}(x)$ described here in the coordinate representation; its dynamics obeys the time dependent Schrödinger equation. Denoting by $\psi(x, t)$ the state at the time $t$ one can write the evolution equations for the free system:

$$
\left\{\begin{array}{l}
i \hbar \frac{\partial}{\partial t} \psi(x, t)=H_{0} \psi(x, t) \\
\psi(x, t=0)=\psi_{0}(x),\left\|\psi_{0}\right\|_{L^{2}\left(\mathbb{R}^{\gamma}\right)}=1
\end{array}\right.
$$

In the presence of external interactions that will be taken here as a control field amplitude $\epsilon(t) \in \mathbb{R}, t \geq 0$ coupled to the system through a time-independent (e.g. dipole) operator $\mu$, the (controlled) dynamical equations read:

$$
\left\{\begin{array}{l}
i \hbar \frac{\partial}{\partial t} \psi_{\epsilon}(x, t)=H_{0} \psi_{\epsilon}(x, t)-\epsilon(t) \mu \psi_{\epsilon}(x, t)=H \psi_{\epsilon}(x, t) \\
\psi_{\epsilon}(x, t=0)=\psi_{0}(x)
\end{array}\right.
$$

Remark 4 For the sake of simplicity we have chosen in this section to treat situations with only one control law present. Extensions to many control laws representing coupling via various other operators are also available (especially for finite dimensional settings); we refer the reader to Ramakrishna et al. (1995); Albertini and D'Alessandro (2001); Turinici et al. (2002) for details.

The $L^{2}$ norm of $\psi_{\epsilon}$ is conserved throughout the evolution:

$$
\left\|\psi_{\epsilon}(x, t)\right\|_{L_{x}^{2}(\mathbb{R} \gamma)}=\left\|\psi_{0}\right\|_{L^{2}(\mathbb{R} \gamma)}, \forall t>0
$$

so the state (or wave-) function $\psi(t)$, evolves on the (complex) unit sphere $S=\left\{\psi \in L^{2}\left(\mathbb{R}^{\gamma}\right):\|\psi\|_{L^{2}\left(\mathbb{R}^{\gamma}\right)}=1\right\}$ according to the Schrödinger equation (9.3) from the initial state $\psi_{0}$ to some final state $\psi(T)$.

The study of the controllability of equation (9.3) is concerned with identifying the set of final states $\psi(T)$ that can be obtained from a given initial state $\psi_{0}$ for all admissible controls. To date, very different results are available for the infinite and finite dimensional settings: while controllability is reasonably well understood for finite dimensional systems, no positive results have been obtained for infinite dimensional systems. 


\section{Infinite dimensional controllability}

Very few results are available concerning the controllability of the equation (9.3) in its infinite dimensional form. Generic negative results have been obtained, as is the following (Thm. 1 from Turinici (2000c); see also Ball et al. (1982); Turinici (2000b)):

Theorem 5 Let $S$ be the complex unit sphere of $L^{2}\left(\mathbb{R}^{\gamma}\right)$. Let $\mu$ be a bounded operator from the Sobolev space $H_{x}^{2}\left(\mathbb{R}^{\gamma}\right)$ to itself and let $H_{0}$ generate a $C^{0}$ semigroup of bounded linear operators on $H_{x}^{2}\left(\mathbb{R}^{\gamma}\right)$. Denote by $\psi_{\epsilon}(x, t)$ the solution of (9.3). Then the set of attainable states from $\psi_{0}$ defined by

$$
\mathcal{A} S=\cup_{T>0}\left\{\psi_{\epsilon}(x, T) ; \epsilon(t) \in L^{2}([0, T])\right\}
$$

is contained in a countable union of compact subsets of $H_{x}^{2}\left(\mathbb{R}^{\gamma}\right)$. In particular its complement with respect to $S: \mathcal{N}=S \backslash \mathcal{A} S$ is everywhere dense on $S$. The same holds true for the complement with respect to $S \cap H_{x}^{2}\left(\mathbb{R}^{\gamma}\right)$.

The theorem implies that for any $\psi_{0} \in H_{x}^{2}\left(\mathbb{R}^{\gamma}\right) \cap S$, within any open set around an arbitrary point $\psi \in H_{x}^{2}\left(\mathbb{R}^{\gamma}\right) \cap S$ there exists a state unreachable from $\psi_{0}$ with $L^{2}$ controls.

Remark 6 The lack of positive controllability results to complement Thm. 5 should be regarded as a failure of available control theory tools to provide insight into controllability rather than as an actual restriction. It is believed that new tools and concepts will make positive results possible, especially since such results are available for the finite dimensional setting (cf. Section 11).

Truncating an infinite-dimensional quantum control problem to a finite-dimensional problem (i.e., so that evolution takes place in a finite dimensional vector space) changes the nature of both the control and Hamiltonian operators and the set of states available as candidate members of reachable sets. The concern is to characterize these effects by asking how a controllability result obtained in a finite-dimensional space relates to the original infinite dimensional problem from which it was derived; there are also inherently finite dimensional quantum systems (as with spins) where the latter consideration does not arise. The controllability of finite dimensional systems is the subject of the next section.

\section{Finite dimensional controllability}

\section{Introduction}

Let $D=\left\{\psi_{i}(x) ; i=1, . ., N\right\}$ be an orthonormal basis for a finite dimensional 
sub-space of $L^{2}\left(\mathbb{R}^{\gamma}\right)$ of interest (for instance the vector space spanned by the first $N$ eigenstates of the internal Hamiltonian $H_{0}$ in Eqn. (9.2)). Denote by $M$ the linear space that $D$ generates, and let $A$ and $B$ be the matrices of the operators $-i H_{0}$ and $-i \mu$ respectively, with respect to this basis. In order to exclude trivial control settings, it is supposed that $[A, B] \neq 0$ (the Lie bracket $[\cdot, \cdot]$ is defined as $[U, V]=U V-V U)$. Note that since $H_{0}$ and $\mu$ are Hermitian operators, the matrices $A$ and $B$ are skew-Hermitian.

Let us denote by $c_{\epsilon}(t)=\left(c_{\epsilon i}(t)\right)_{i=1}^{N}$ the coefficients of $\psi_{i}(x)$ in the expansion of the evolving state $\psi(t, x)=\sum_{i=1}^{N} c_{\epsilon i}(t) \psi_{i}(x)$; then equation (9.3) reads

$$
\begin{gathered}
\left\{\begin{array}{l}
\frac{d}{d t} c_{\epsilon}(t)=A c_{\epsilon}(t)-\epsilon(t) B c_{\epsilon}(t) \\
c_{\epsilon}(t=0)=c_{0}
\end{array}\right. \\
c_{0}=\left(c_{0 i}\right)_{i=1}^{N}, c_{0 i}=<\psi_{0}, \psi_{i}>_{L^{2}\left(\mathbb{R}^{\gamma}\right)}
\end{gathered}
$$

(where atomic units are used i.e. we set $\hbar=1$ ). Note as in Eqn. (9.4) that the system (11.1) evolves on the unit sphere $S_{N}$ of $L^{2}\left(\mathbb{R}^{\gamma}\right) \cap M$ which reads,

$$
\sum_{i=1}^{N}\left|c_{\epsilon i}(t)\right|^{2}=1, \quad \forall t \geq 0
$$

Note also that the solution $c_{\epsilon}(t)$ of Eqn. (11.1) can be written

$$
c_{\epsilon}(t)=U_{\epsilon}(t) c_{0}
$$

where the time evolution operator $U_{\epsilon}(t)$ is the solution of the following

$$
\left\{\begin{array}{l}
\frac{d}{d t} U_{\epsilon}(t)=A U_{\epsilon}(t)-\epsilon(t) B U_{\epsilon}(t) \\
U_{\epsilon}(t=0)=I_{N \times N}
\end{array}\right.
$$

The matrix $U(t)$ evolves in the Lie group of unitary matrices $U(N)$, or, if both the matrices $A$ and $B$ have zero trace, in the Lie group of special unitary matrices $S U(N)$. Equation (11.5) also prescribes the evolution of the density matrix operator: if the system starts in the mixture of states represented by the density operator $\rho_{0}$ then its evolution is given by the formula

$$
\rho(t)=U(t) \rho_{0} U(t)^{\dagger}
$$

where for any matrix $X$ we denote by $X^{\dagger}$ its transpose-conjugate. 
Remark 7 Note that since $U(t)$ is unitary $\rho(t)$ has the same eigenvalues as $\rho(0)$.

We now introduce:

Definition 8 Denote by $\mathcal{U}$ the set of all admissible control laws $\epsilon(t)$. The system described by the state $c_{\epsilon}(t)$ is called state-controllable if for any $c_{i}, c_{f} \in$ $S_{N}$ there exists $0<\tau<\infty$ and $\epsilon \in \mathcal{U}$ such that $c_{\epsilon}(t=\tau)=c_{f}$, where $c(t)$ satisfies (9.3) with $c_{\epsilon}(t=0)=c_{i}$.

Within the density matrix formulation the relevant definition of controllability becomes:

Definition 9 The system described by the evolution of the density operator $\rho(t)$ satisfying the Eqns. (11.5)-(11.6) is called density-matrix-controllable if for any two density operators $\rho_{i}$ and $\rho_{f}$ compatible in the sense that there exists an unitary matrix $U$ such that $\rho_{f}=U \rho_{i} U^{\dagger}$ there exists a control law $\epsilon(t) \in \mathcal{U}$ such that given the initial condition $\rho(0)=\rho_{i}$ then $\rho(\tau)=\rho_{f}$ for some finite time $\tau$.

Remark 10 Because pure states my be represented by density matrices a system that is density-matrix-controllable is also state-controllable.

\section{Lie group methods}

Let us introduce the following

Definition 11 A subset $\mathcal{T}$ of $U(N)$ (or $S U(N)$ ) is said to be transitive (to act transitively) on the sphere $S_{N}$ if for any $c_{i}, c_{f} \in S_{N}$ there exists $g \in \mathcal{T}$ such that $c_{f}=g c_{i}$.

With this definition and considering Eqn. (11.4) it follows that

- state-controllability of the wavefunction as in Definition 8 is equivalent to requiring that the set of all matrices attainable from identity $\left\{U_{\epsilon}(t) ; 0 \leq\right.$ $t \leq \infty ; \epsilon \in \mathcal{U}, U$ verify (11.5)\} be transitive on the sphere $S_{N}$, while

- density-matrix-controllability is equivalent to requiring that the set of all matrices attainable from identity be at least $S U(N)$.

Remarkable examples of transitive subgroups of $U(N)$ are $U(N)$ itself, $S U(N)$ and, when $N$ is even, the symplectic matrices $S p(N / 2)$. It can be proven (see Albertini and D'Alessandro (2001)) that, except for some special values of $N$ and up to an isomorphism, these are the only transitive subgroups arising in quantum control.

The important result that turns this remark into a powerful tool for studying the controllability of bilinear systems is that the set of all matrices attainable 
from the identity via (11.5) is given by the connected Lie subgroup $e^{L}$ of the Lie algebra $L$ generated by $A$ and $B$ (when this Lie group is compact) (cf. Ramakrishna et al. (1995); Albertini and D'Alessandro (2001)). The group $e^{L}$ is called the dynamical Lie group of the system.

Taking $\mathcal{U}$ to be the set of all piecewise continuous functions (unconstrained in magnitude) yields the following result:

Theorem 12 (Ramakrishna et al. (1995)) A sufficient condition for the density-matrix- (thus state-) controllability of the quantum system in equation (11.1) is that the Lie algebra $L$ generated by $A$ and $B$ has dimension $N^{2}$ (as a vector space over the real numbers).

Furthermore, if both $A$ and $B$ are traceless then a sufficient condition for the density-matrix- (thus state-) controllability of quantum system is that the Lie algebra $L$ has dimension $N^{2}-1$.

A following result builds on Thm. 12 to gives the necessary and sufficient condition of state controllability:

Theorem 13 (Albertini and D'Alessandro (2001)) The system is state controllable if and only if the Lie algebra $L$ generated by $A$ and $B$ is isomorphic (conjugate) to $\operatorname{sp}\left(\frac{N}{2}\right)$ or to $s u(N)$, if the dimension $N$ is even, or to $s u(N)$, if the dimension $N$ is odd (with or without the $i I$, where $I$ is the identity matrix).

Theorem 12 lends itself to algorithmic (numerical) implementation: as soon as the matrices $A$ and $B$ that characterize the system are given, one can compute (numerically for instance) the Lie algebra they generate and obtain its dimension. However, except for small systems, this test becomes rapidly very computationally expensive; additional results are therefore required in order to shed some light on the relationship between controllability and the structure of the $A$ and $B$ matrices. Two studies in this direction are available: one, from Schirmer et al. (2001); Fu et al. (2001) is presented in this section and the second, the "connectivity graph" approach, is described in the next section (cf. Turinici (2000c); Turinici and Rabitz (2001a,b); Turinici (2000b,a)).

When the basis $D=\left\{\psi_{i}(x) ; i=1, . ., N\right\}$ is composed of eigenstates of the internal Hamiltonian $H_{0}$ the matrix $A$ is diagonal with purely imaginary elements $-i E_{k}$, where $E_{k}$ are the eigenvalues of $H_{0}, k=1, \ldots, N$. Let $\delta_{k}=$ $E_{k}-E_{k+1}$ for $n=1, \cdots, N-1$.

Theorem 14 (Schirmer et al. (2001)) Suppose that the matrix B of the interaction operator $-i \mu$ with respect to the basis $D$ is such that $B_{k, l}=0$ for $|k-l| \neq 1$ and $B_{k, l} \neq 0$ for $|k-l|=1, k, l=1, \ldots, N$. Then if either

$1 / \delta_{1} \neq 0$ and $\delta_{k} \neq \delta_{1}$ for $k=1, \ldots, N-1$, or 
2/ $\delta_{N-1} \neq 0$ and $\delta_{N-1} \neq \delta_{k}$ for $k=1, \ldots, N-1$

the dynamical Lie group of the system $A-\epsilon(t) B$ with $A$ and $B$ as in equation (11.5) is at least $S U(N)$. If in addition $\operatorname{Tr} A \neq 0$ then the dynamical Lie group is $U(N)$. In both cases the system is density-matrix- (thus state-) controllable.

Remark 15 Although the hypothesis of the theorem above are somewhat strong, it allows for the determination of controllability using only generic properties of the system under study, i.e. without the need to know exactly the matrices $A$ and $B$. We will see later in this section (see Remark 20) other results concerning the controllability of the wavefunction that do not require precise evaluation of the $A$ and $B$ matrices.

While the results above hold in the case that the control field amplitudes are not bounded, an open question suggested in this work is the extension of the result to stricter (and more realistic) admissibility conditions: can the Lie algebraic controllability conditions (e.g. Ramakrishna et al. (1995)) be extended to treat the case where both the amplitude and the frequency of the control field are bounded from above and below? This issue has practical significance as it prescribes real laboratory conditions.

Remark 16 Other open problems refer to the controllability within the product state space of the coupled Schrödinger-Maxwell equations for optically dense media. While the Schrödinger-Maxwell system has a product state space representing both $\rho(t)$ (the density operator) and $\epsilon(t)$, expectation values depend only on $\rho(t)$, which is the usual focus of controllability studies. This inspires the question: in what, if any, cases is it possible for the SchrödingerMaxwell system to be controllable in the state space of the quantum state but possibly not controllable in that of the electric field, or vice-versa? The latter case of controlling the electric field is of importance in the allied subject of optical field propagation (e.g., Xia et al. (1997)).

Remark 17 To ease the assessment of controllability using the results of this section, an automatic tool that allows computation of the dimension of the Lie algebra generated by several (skew-Hermitian) matrices is available freely on the Internet (cf. Turinici and Schirmer (2001)).

\section{Controllability analysis via the connectivity graph}

This section seeks to address controllability from an analysis of the kinematic structure of the Hamiltonian. Suppose that the basis $D=\left\{\psi_{i}(x) ; i=1, . ., N\right\}$ is composed of eigenstates of the internal Hamiltonian $H_{0}$, so that the matrix $A$ is diagonal with purely imaginary elements $-i E_{k}$, where $E_{k}$ are the eigenvalues of $H_{0}, k=1, \ldots, N$ and that the diagonal elements of the matrix $B$ are all zero 
(this is often the case in practice). We obtain the following structure:

$$
A=-i\left(\begin{array}{cccc}
E_{1} & & & 0 \\
& E_{2} & & \\
& & \ddots & \\
& & & \\
0 & & & E_{n}
\end{array}\right) ; B=-i\left(\begin{array}{cccc}
0 & b_{12} & \ldots & b_{1 N} \\
b_{12}^{*} & 0 & b_{i j} & \vdots \\
\vdots & b_{i j}^{*} & \ddots & \\
b_{1 N}^{*} & \ldots & & 0
\end{array}\right) \text {. }
$$

Assume moreover that no degenerate transitions are present, i.e.

$$
\left|E_{i}-E_{j}\right| \neq\left|E_{k}-E_{l}\right|, i, j, k, l=1, \ldots, n, i \neq j, k \neq l,\{i, j\} \neq\{k, l\} .
$$

The connectivity amongst the states $\left\{\psi_{i} ; i=1, \ldots, n\right\}$ provided by the elements $b_{i j}, i, j=1, \ldots, n$ is central to issues of controllability. The structure in $B$ can be conveniently expressed graphically by introducing a graph $G=(V, E)$ (see Christofides (1975) for an introduction to graph theory): let every state be a vertex (node) of the graph $G$ so that the set of vertices $V=\left\{\psi_{1}, \ldots, \psi_{N}\right\}$, and let there be edges between every pair of nodes $\psi_{i}$ and $\psi_{j}$ with $b_{i j} \neq 0$ so that the set of edges $E=\left\{\left(\psi_{i}, \psi_{i}\right) ; b_{i j} \neq 0\right\}$. Two states $\psi_{i}$ and $\psi_{j}$ are said to be connected by a path if there exists a connected set of edges starting in $\psi_{i}$ and ending in $\psi_{j}$. The graph $G$ is called connected if there exists a path between every pair of vertices.

Remark 18 Note that $G$ being connected does not imply that any two states are necessarily directly connected (i.e. with a direct edge). One such example is the (connected) graph in Figure 1 associated with the system

$$
A=-i\left(\begin{array}{rrrr}
1.1 & & & 0 \\
& 2.3 & & \\
& 3.05 & \\
& & & 4.6
\end{array}\right) ; B=-i\left(\begin{array}{llll}
0 & 0 & 1 & 0 \\
0 & 0 & 1 & 0 \\
1 & 1 & 0 & 1 \\
0 & 0 & 1 & 0
\end{array}\right)
$$

These definitions allow formulating the following result:

Theorem 19 Turinici and Rabitz (2001a,b) Suppose that the graph $G$ is connected and that the transitions of the internal Hamiltonian are non-degenerate in the sense of Eqn. (11.7). Then the system in (11.1) is state-controllable.

Remark 20 The conditions of connectivity and non-degenerate transition frequencies involve only the eigenvalues of $H_{0}$ and the coefficients of $B$ (which 


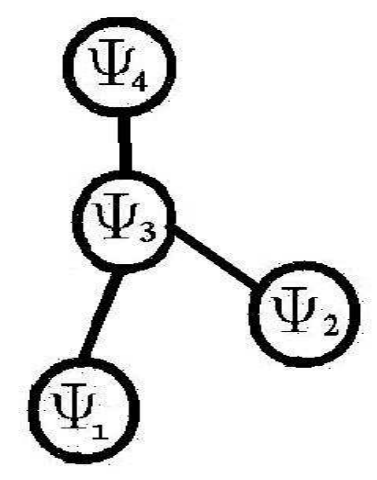

Fig. 1. The (connected) graph associated with the $B$ matrix of the system in Eqn. (11.8). Note that no direct path exits between, e.g. $\psi_{1}$ and $\psi_{4}$.

depend only upon which elements of $B$ are non-zero, rather than their particular values) allowing conclusions about the controllability of the system in Eqn. (11.1) even in the absence of quantitative information about this system.

Remark 21 Controllability generally does not provide an actual control law that will achieve an objective of the form (4.1), but simply implies that at least one such control law exists. The proof of controllability in Turinici (2000c) establishes an algorithm which constructively generates control laws for objective operators of the form $O_{i}=\left|\psi_{i}\right\rangle\left\langle\psi_{i}\right|$ in terms of sinusoidal electric fields of different fixed frequencies. Note that in this situation, the control objectives are populations of quantum states (e.g., $\left.\left|\left\langle\psi \mid \psi_{i}\right\rangle\right|^{2}\right)$.

This latter work, along with other work on special cases of constructive quantum control (e.g. Harel and Akulin (1999); Ramakrishna et. al. (2000)), invites the question: can general constructive control solutions be developed for the quantum controllability of a broad class of objective expectation values? While the explicit construction of control solutions is generally an area of active research in control theory (Nijmeiher and van der Schaft (1990); Sepulchre et al. (1998)), the case of quantum controllability of expectation values reduces this task to the specific structure of Schrödinger's equation that may be amenable to attack.

Remark 22 The analysis of controllability in terms of state functions $\psi(t)$ reaches beyond what is necessary physically, as realistic objectives are expectation values of observable operators (c.f. Eqn. (4.1)). Since these quantities involve integrals of state functions, their control should generally be less demanding than that of the state itself. However, the quadratically nonlinear nature of the expectation values adds a level of additional complexity to the problem of determining controllability.

Having seen positive results for finite-dimensional wavefunction controllability we now investigate what phenomena prevent this controllability. Even if a final 
answer does not yet exist, available evidence suggests that conservation laws are responsible for the loss of controllability. Let us consider, as in Turinici and Rabitz (2001a), the following simple 3-level system:

$$
A=\left(\begin{array}{lll}
1 & 0 & 0 \\
0 & 2 & 0 \\
0 & 0 & 3
\end{array}\right), B=\left(\begin{array}{lll}
0 & 1 & 0 \\
1 & 0 & 1 \\
0 & 1 & 0
\end{array}\right),
$$

and the corresponding evolution equations

$$
\begin{aligned}
& i \frac{d}{d t} c_{\epsilon 1}(t)=c_{\epsilon 1}(t)+\epsilon(t) c_{\epsilon 2}(t) \\
& i \frac{d}{d t} c_{\epsilon 2}(t)=2 c_{\epsilon 2}(t)+\epsilon(t) c_{\epsilon 1}(t)+\epsilon(t) c_{\epsilon 3}(t) \\
& i \frac{d}{d t} c_{\epsilon 3}(t)=3 c_{\epsilon 3}(t)+\epsilon(t) c_{\epsilon 2}(t)
\end{aligned}
$$

This system has degenerate transitions as $E_{2}-E_{1}=E_{3}-E_{2}$. Upon closer examination, a "hidden symmetry" is found for this system: more precisely it is easy to prove that for any $t>0$ and $\epsilon(t) \in L^{2}([0, t])$ :

$$
\left|c_{\epsilon 1}(t) c_{\epsilon 3}(t)-\frac{c_{\epsilon 2}(t)^{2}}{2}\right|=\left|c_{\epsilon 1}(0) c_{\epsilon 3}(0)-\frac{c_{\epsilon 2}(0)^{2}}{2}\right| .
$$

Therefore, any $\psi(t)=\sum_{i=1}^{3} c_{\epsilon i}(t) \psi_{i}(x)$ that is reachable from $\psi(0)$ with $\psi(0)=$ $\sum_{i=1}^{3} c_{\epsilon i}(0) \psi_{i}(x)$ must satisfy the constraint (11.10). Let us consider a simple numerical example: suppose that the initial state is the ground state $\psi_{1}$ and the target is the first excited state $\psi_{2}$. We obtain for $\psi_{1}:\left|c_{\epsilon 1}(0) c_{\epsilon 3}(0)-\frac{c_{\epsilon 2}(0)^{2}}{2}\right|=$ $\left|1 \cdot 0-\frac{0^{2}}{2}\right|=0$ and for $\psi_{2}:\left|c_{\epsilon 1}(t) c_{\epsilon 3}(t)-\frac{c_{\epsilon 2}(t)^{2}}{2}\right|=\left|0 \cdot 0-\frac{1^{2}}{2}\right|=\frac{1}{2}$. Since the two quantities are different, $\psi_{2}$ is not reachable from $\psi_{1}$ and therefore the system is not controllable, despite the fact that the connectivity assumption is satisfied.

The detailed analysis of the case $N=3$ and a result that was communicated to us (cf. Ramakrishna (2000)) suggest the conjecture that for general finitedimensional systems, as long as no conservation laws appear (besides $L^{2}$ norm conservation) the system is controllable. This statement, if true, would have the merit of giving a result only in terms of the physical properties of the system under consideration (and independent of the mathematical transcription of the precise control situation).

Remark 23 For the density matrix formulation, kinematical constraints on the controllability of systems in mixed states have been established (e.g. Girardeau et al. $(1998,1997))$, based on the eigenvalues of $\rho(0)$ and those of the objective operator. 


\section{Independent systems controllability and discrimination issues}

This section introduces controllability results for independent quantum systems. Most of the text draws from Turinici et al. (2002).

Successful control may be expressed as a matter of high quality discrimination, whereby the control field steers the evolving quantum system dynamics out the desired channel, while diminishing competitive flux into other undesirable channels. A potential application of quantum control techniques is to the detection of specific molecules amongst others of similar chemical/physical characteristics. We call this procedure coherent molecular discrimination. Cases of special interest for discrimination include large polyatomic molecules of similar chemical nature, whose spectra can often mask each other.

Much work needs to be done to explore and develop the concept of coherent molecular discrimination, and a basic step in this direction is to establish the criteria for independently and simultaneously controlling the dynamics of several molecular species with the same control field. Discrimination of multiple molecules is a special case of the controllability concept, where the full system consists of a set of subsystems (i.e., molecules of different type). In the simplest circumstances, the molecules may be taken as independent and noninteracting, such that the initial state $\psi(0)$ is a product $\psi(0)=\prod_{\ell=1}^{L} \psi_{\ell}(0)$ of states $\psi_{\ell}(0)$ for each of the $L \geq 2$ molecular species. Full controllability would correspond to the ability to simultaneously and arbitrarily steer about each

of initial states $\psi_{\ell}(0)$ to predefined targets $\psi_{\ell}(T)=\psi_{\ell}^{\text {target }}$ under the influence of a single control laser electric field $\epsilon(t)$, where each molecule evolves under a separate Schrödinger equation

$$
i \hbar \frac{\partial}{\partial t} \psi_{\ell}(t)=\left[H_{0}^{\ell}-\mu^{\ell} \cdot \epsilon(t)\right] \psi_{\ell}(t)
$$

Here, $H_{0}^{\ell}$ and $\mu^{\ell}$, respectively, are the free Hamiltonian and interaction operator (dipole) of the $\ell$-th molecule. Other milder controllability criteria might also be specified.

The purpose of this section is to state theoretical criteria for the controllability of an ensemble of $L$ separate quantum systems in the presence of a single electric field $\epsilon(t)$. The criteria will refer to a finite dimensional setting where, for each $\ell, 1 \leq \ell \leq L$, the Hamiltonian $H_{0}^{\ell}$ and dipole operator $\mu^{\ell}$ are expressed with respect to an eigenbasis of the internal Hamiltonians, as is often the case in applications. More precisely, let $D^{\ell}=\left\{\psi_{i}^{\ell}(x) ; i=1, . ., N_{\ell}\right\}$ be the set of the first $N_{\ell}, N_{\ell} \geq 3$ eigenstates of the possibly infinite dimensional Hamiltonian $H_{0}^{\ell}$, and let $A^{\ell}$ and $B^{\ell}$ be the matrices of the operators $-i H_{0}^{\ell}$ and $-i \mu^{\ell}$ respectively, with respect to this base. In order to exclude a trivial loss of controllability, it is supposed that $\left[A^{\ell}, B^{\ell}\right] \neq 0, \ell=1, \ldots, L$. From the 
definition of the basis $D^{\ell}$ and the fact that $H_{0}^{\ell}$ and $\mu^{\ell}$ are Hermitian operators, it follows that each $A^{\ell}$ is diagonal with purely imaginary elements and each $B^{\ell}$ is skew-Hermitian. We will suppose moreover that

For any $\ell=1, \ldots, L: A^{\ell}$ has nonzero trace and $B^{\ell}$ has zero trace. (11.12)

With this notation, the wavefunction of the $\ell$-th system can be written as $\psi_{\ell}(t)=\sum_{i=1}^{N_{\ell}} c_{i}^{\ell}(t) \psi_{i}^{\ell}$. The total eigenfunction $\prod_{\ell=1}^{L} \psi_{\ell}(t)$ will be represented as a column vector $c(t)=\left(c_{1}^{1}(t), \ldots, c_{N_{1}}^{1}(t), \ldots, c_{1}^{L}(t), \ldots, c_{N_{L}}^{L}(t)\right)^{T}$. Denote $N=$ $\sum_{\ell=1}^{L} N_{\ell}, A$ be the $N \times N$ skew-Hermitian block-diagonal matrix obtained from $A^{\ell}, \ell=1, \ldots, L$ and $B$ be the skew-Hermitian block-diagonal matrix obtained from $B^{\ell}, \ell=1, \ldots, L$ :

$$
A=\left(\begin{array}{cccc}
A^{1} & 0 & \ldots & 0 \\
0 & A^{2} & \ldots & 0 \\
\vdots & \vdots & \ddots & \vdots \\
0 & 0 & \ldots & A^{L}
\end{array}\right), B=\left(\begin{array}{cccc}
B^{1} & 0 & \ldots & 0 \\
0 & B^{2} & \ldots & 0 \\
\vdots & \vdots & \ddots & \vdots \\
0 & 0 & \ldots & B^{L}
\end{array}\right)
$$

With the "atomic units" convention $\hbar=1$, the dynamical equations read:

$$
\frac{d}{d t} c(t)=A c(t)+\epsilon(t) B c(t), c(0)=c_{0} .
$$

Recalling that each individual wavefunction $\psi_{\ell}(t)=\sum_{i=1}^{N_{\ell}} c_{i}^{\ell}(t) \psi_{i}^{\ell}$ is $L^{2}$ normalized to one, we obtain:

$$
\sum_{i=1}^{N_{\ell}}\left|c_{i}^{\ell}(t)\right|^{2}=1, \forall t \geq 0, \forall \ell=1, \ldots, L .
$$

Let $\mathcal{S}_{\mathbb{C}}^{k-1}$ be the complex unit sphere of $\mathbb{C}^{k}$. Then equation (11.15) gives:

$$
c(t) \in \mathcal{S}=\prod_{\ell=1}^{L} \mathcal{S}_{\mathbb{C}}^{N_{\ell}-1}, \forall t \geq 0 .
$$

Define the admissible control set $\mathcal{U}$ as the set of all piecewise continuous functions $\epsilon(t)$. For every $\epsilon \in \mathcal{U}$ Eqn. (11.14) has an (unique) solution for all $t \geq 0$. The system $\left(\left(A^{\ell}, B^{\ell}\right)_{\ell=1}^{L}, \mathcal{U}\right)$ is said to be controllable if for any $c_{i}, c_{f} \in \mathcal{S}$ there exists an $t_{f} \geq 0$ (possibly depending on $c_{i}, c_{f}$ ) and $\epsilon(t) \in \mathcal{U}$ such that the solution of (11.14) with initial data $c(0)=c_{i}$ satisfy $c\left(t_{f}\right)=c_{f}$. Of course, in order for the system $\left(\left(A^{\ell}, B^{\ell}\right)_{\ell=1}^{L}, \mathcal{U}\right)$ to be controllable each component system $\left(A^{\ell}, B^{\ell}, \mathcal{U}\right), \ell=1, \ldots, N$ taken independently has to be controllable. However, requiring that all systems be controllable at the same time and 
with the same laser field is a more demanding condition. To illustrate this statement, we will consider the simple case of two $(L=2)$ three-level systems $\left(N_{1}=3, N_{2}=3\right)$ of Turinici et al. (2002):

$$
A^{1}=A^{2}=-i \cdot\left(\begin{array}{lll}
1 & 0 & 0 \\
0 & 2 & 0 \\
0 & 0 & 5
\end{array}\right), B^{1}=-i \cdot\left(\begin{array}{lll}
0 & 1 & 0 \\
1 & 0 & 2 \\
0 & 2 & 0
\end{array}\right), B^{2}=-B^{1}
$$

Each system $A^{1}, B^{1}$ and $A^{2}, B^{2}$ is controllable, as can be checked by the Lie algebra criterion of Ramakrishna et al. (1995) (the dimension of the Lie algebra is found to be 9 ).

However, denoting by $\left(c_{1}^{1}, c_{2}^{1}, c_{3}^{1}\right)$ and by $\left(c_{1}^{2}, c_{2}^{2}, c_{3}^{2}\right)$ the coefficients of the wavefunction of the first system and of the second system respectively, we obtain the following dynamical invariant (conservation law):

$$
L(t)=\overline{c_{1}^{1}(t)} c_{1}^{2}(t)+\overline{c_{3}^{1}(t)} c_{3}^{2}(t)-\overline{c_{2}^{1}(t)} c_{2}^{2}(t)=\text { constant }, \forall \epsilon \in \mathcal{U}
$$

The presence of this conservation law implies that the system is not controllable. For instance, starting with both systems in the ground state

$$
\left(c_{1}^{1}(0), c_{2}^{1}(0), c_{3}^{1}(0)\right)=\left(c_{1}^{2}(0), c_{2}^{2}(0), c_{3}^{2}(0)\right)=(1,0,0)
$$

one cannot steer both to their respective first excited state

$$
\left(c_{1}^{1}(T), c_{2}^{1}(T), c_{3}^{1}(T)\right)=\left(c_{1}^{2}(T), c_{2}^{2}(T), c_{3}^{2}(T)\right)=(0,1,0)
$$

since in the ground states the dynamical invariant takes the value $L(0)=$ $1+0-0=1$ while in the first excited states the value is $L(T)=0+0-1=-1$.

Defining the set of attainable states

$$
\mathcal{A}\left(c_{0}, T\right)=\{c(t) ; c(t) \text { solution of }(11.14), t \in[0, T], u \in \mathcal{U}\}
$$

the system is controllable if (and only if) for any $c_{0} \in \mathcal{S}$ the set of points attainable from $c_{0}: \bigcup_{t \geq 0} \mathcal{A}\left(c_{0}, t\right)$ equals $\mathcal{S}$.

We are now ready to state the following controllability result:

Theorem 24 (Turinici et al. (2002)) If the dimension (computed over the scalar field $\mathbb{R})$ of the Lie algebra $\mathcal{L}(A, B)$ generated by $A$ and $B$ equals $1+$ $\sum_{\ell=1}^{L}\left(N_{\ell}^{2}-1\right)$ then the system $\left(\left(A^{\ell}, B^{\ell}\right)_{\ell=1}^{L}, \mathcal{U}\right)$ is controllable. Moreover, when 


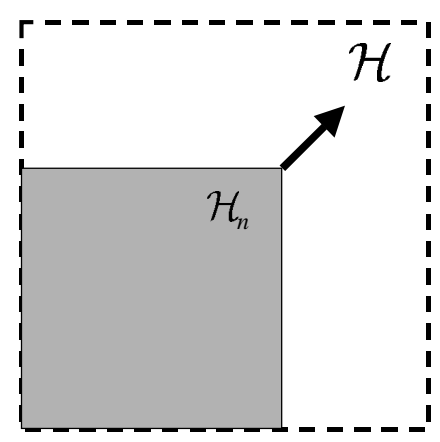

a)

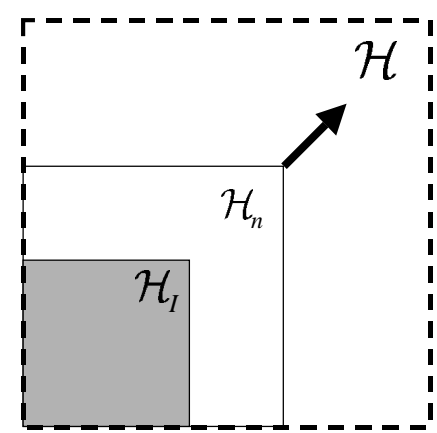

b)

Fig. 2. Pictorial representation of the truncation problem. In both cases, controllability is of interest within the shaded subspace as the dimension of the truncated space $H_{n}$ increases to infinity.

the system is controllable, there exists a time $T>0$ such that all targets can be attained before or at time $T$, i.e. for any $c_{0} \in \mathcal{S}, \mathcal{A}\left(c_{0}, T\right)=\mathcal{S}$.

We refer to Li et al. (2002); Turinici et al. (2002) for more general results where the hypotheses of Eqn. (11.12) are not satisfied or when multiple $s>1$ external fields are considered (which can be expressed by introducing multiple dipole moment operators $\left.\mu_{i}^{\ell}, i=1, \ldots, s\right)$.

\section{Truncations}

Little is known about the relationship between the controllability of the finite dimensional systems and that of infinite dimensional systems. We will discuss in this section some of the interesting aspects of this interplay through a list of open problems and questions.

Consider a quantum system that is controllable when its (truncated) equations of motion are expressed with respect to a particular $n$-dimensional basis which spans a finite dimensional space $\mathcal{H}_{n}$. According to Theorem 5 , for every initial condition there must emerge a dense set of unreachable states in the limit $n$ tends to infinity (depicted in Figure 2(a)), assuming that the limiting process is well defined. In other words, for $n \rightarrow \infty$ the system may become uncontrollable in the strict sense defined above. This limit suggests the question: how does the controllability of a sequence of finite but increasingly higher dimensional quantum systems relate to the controllability of the corresponding infinite-dimensional quantum system in the limit $n \rightarrow \infty$ (if this limiting process exists)? How are the sets of unreachable states that emerge in this limit characterized?

The analysis implied in the above questions can be subtle, as evident from a 
simple illustration involving the emergence or disappearance of unreachable states under finite increases in the dimensionality of $\mathcal{H}_{n}$. For example, in hydrogenic atoms the transitions due to emission or absorption of photons must satisfy the selection rules $\Delta l= \pm 1$ and $\Delta m= \pm 1$ or 0 . If the step $\mathcal{H}_{n} \rightarrow \mathcal{H}_{n+1}$ of the limiting process adds a basis function to which there does not exist a sequence of allowed transitions from some function $\psi_{1}$ in $\mathcal{H}_{n}$, the additional dimension has caused a loss of system controllability. The converse situation may also arise where the additional basis function provides a "missing pathway" between states that were mutually unreachable in $\mathcal{H}_{n}$ : in this case, the step $\mathcal{H}_{n} \rightarrow \mathcal{H}_{n+1}$ might cause an uncontrollable system to become controllable. It is an open question in quantum controllability to understand how such stepwise processes may be interpreted in the infinite limit.

Now consider the related issue of controllability within a "subspace of interest" $\mathcal{H}_{I}$ that is contained within $\mathcal{H}_{n}$ (as depicted in Figure $2(\mathrm{~b})$ ). Let $\mathcal{H}_{I}$ be spanned by the first $I$ elements of the set of basis functions $\left\{\psi_{i}: i=1 \ldots n\right\}$ spanning $\mathcal{H}_{n}$. Definition 8 may be modified to restrict analysis to the subspace of interest: controllability will be taken to mean that a system is controllable if the system can be steered between any two states $\psi_{1}^{I}$ and $\psi_{2}^{I}$ in $S \cap \mathcal{H}_{I}$. Controllability may be described as stationary within $\mathcal{H}_{I}$ if it remains unchanged as individual dimensions are added in any order to $\mathcal{H}_{n}$ until (if it exists) the limit $\lim _{n \rightarrow \infty, n \geq I} \mathcal{H}_{n}=\mathcal{H}$, is obtained. It is not known what characteristics of the Hamiltonian $H_{0}$, the dipole or other coupling coefficients, and the spaces $\mathcal{H}_{I} \subset \mathcal{H}_{n} \subset \mathcal{H}$ are required for stationary controllability within $\mathcal{H}_{I}$.

The discussion above does not address the effects on the evolution of states within the truncated space $\mathcal{H}_{n}$ arising from states that lie outside of $\mathcal{H}_{n}$. This consideration also has practical consequences. For example, suppose that controllability is satisfied within $\mathcal{H}_{n}$ or within $\mathcal{H}_{I}$ for some $\mathcal{H}_{n}$. A realizable laboratory control might inadvertently also access states lying outside of $\mathcal{H}_{n}$ which might even lift the controllability in the desired subspace. Techniques from optimal control theory would be the desirable way to handle the discovery of practical fields best satisfying the assumptions under an associated controllability analysis. Following upon the latter discussion, a new class of problems is introduced if a term is added to the Schrödinger equation to represent the interaction of the remainder states that are not explicitly modeled lying in $\mathcal{H} \backslash \mathcal{H}_{n}$. One such term introduced in Beumee and Rabitz (1992), c.f. also Sontag (1998), is a $n$-dimensional disturbance vector $w$ :

$$
\frac{\partial \psi}{\partial t}=\left[H_{0}+C(t)\right] \psi+w
$$

where $\psi \in \mathcal{H}_{n}$. The form and magnitude of the disturbance term $w$ is problemdependent and assumed to be given a priori, and generally leads to a nonunitary evolution for $\psi(t)$. In min-max optimal control theory (c.f. Section 15), w 
is selected to maximize the disruptive effect of the energy-bounded disturbance (e.g., fluctuations in the laboratory environment and apparatus). In another general context, $w$ could represent coupling to a bath external to the dynamics described by $H_{0}+C(t)$. This type of coupling is important for considerations of dynamical cooling (see Bartana and Kosloff $(1993,1997)$; Tannor and Bartana (1999); Tannor et al. (1999)) in the analogous density matrix formulation. It is not yet clear what general models of dynamics exterior to $\mathcal{H}_{n} \subset \mathcal{H}$ can cause controllable systems to become uncontrollable and vice-versa.

\section{Chapter 4. Quantum control algorithms}

As analytical solutions to the quantum optimal control equations cannot generally be found, iterative numerical algorithms must be employed. The timedependent Schrödinger equation in multiple spatial variables is computationally very expensive to solve, and, although there appear to be many opportunities to develop special control design approximations, and while some work has already been done (e.g. Rice and Zhao (2000)), there is room for much development.

Remark 25 Since the Schrödinger equation must be solved at least once (and generally many times) in most optimal control and Hamiltonian identification methods, the numerical evolution of quantum systems with many degrees of freedom must be approximated in some fashion before control design techniques can be applied. Along these lines, broad classes of quantum dynamics approximations have been developed, and in principle any of them could be applied to quantum control design. While attempting to attain designs, it is worth investigating the effects on controllability of replacing the Schrödinger equation with its various quantum dynamical approximations. Significant influence of a dynamical approximation upon a system's controllability could have serious consequences for the reliability of any resultant control designs based on the approximation.

A very important feature of quantum control is the intricate relationship between theory and the laboratory implementations, where an optimization method, usually an evolutionary (e.g. genetic) algorithm, is used to drive the experimental work which, in its turn, feeds back the optimization algorithm with necessary data. Reflecting this connection, both numerical and experimental paradigms are presented in this section, the first in Section 14 (extending with connected topics through Section 16) and the latter in Section 17. Common to both, the construction of the cost functionals is the object of the next section. 


\section{Optimal control cost functional formulation}

As explained in Section 5, the first step in formulating the general quantum optimal control problem is to define a "cost functional" whose minimization represents the balanced achievement of control and possibly other objectives. This cost functional is given by

$$
J=\sum_{k} J_{k}(C(\psi(t) ; t \in[0, T]))
$$

where the goal is to minimize (or maximize, as appropriately) $J$ with respect to $C(t)$. While the specific form of the cost functional is flexible and problemdependent, a term $J_{1}$ that addresses the achievement of the $N_{O}$ optimal control objectives is always included:

$$
J_{1}=\sum_{j=1}^{N_{O}} \sum_{l=1}^{L_{j}}\left\{\begin{array}{c}
\int_{\tau_{j}^{l}} W_{1, j}^{l}\left[\left\langle O_{j}(t)\right\rangle-\tilde{O}_{j}(t)\right]^{\gamma} d t, \text { if } \tau_{j}^{l} \text { is an interval } \\
W_{1, j}^{l}\left[\left\langle O_{j}\left(\tau_{j}^{l}\right)\right\rangle-\tilde{O}_{j}\left(\tau_{j}^{l}\right)\right]^{\gamma}, \text { if } \tau_{j}^{l} \text { is a discrete time }
\end{array}\right.
$$

Here the $W_{1, j}^{l}$ are positive design weights assigned to each of the objectives. The particular case $N_{O}=L_{1}=\gamma=W_{1,1}^{1}=1, \tau_{1}^{1}=T$ and $\tilde{O}_{1}(T)=0$ has already been seen (cf. Eqn. (5.1)) to give rise to a term of the form $J_{1}=\left\langle\psi(T)\left|O_{1}\right| \psi(T)\right\rangle$.

For physically realistic control laws, the energy of the laboratory/molecular interaction must be bounded. This criteria is often included by adding the term

$$
J_{2}=\int_{0}^{T} W_{2}(t) \epsilon(t)^{2} d t
$$

to the cost functional, which effectively limits the total electric field fluence. Here, $W_{2}(t)$ determines the time-dependent relative importance of minimizing the fluence. Note that the term $J_{2}$ does not prevent $\epsilon(t)$ from being large in some small interval of time, although a cost on the local magnitude at any time could be introduced for this purpose.

Penalty terms may also be included, causing the minimization of the expectation values of $N_{O^{\prime}}$ "undesirable" operators $O_{j}^{\prime}$ at the corresponding times $\tau_{j}^{\prime l}:$

$$
J_{3}=\sum_{j=1}^{N_{O}^{\prime}} \sum_{l=1}^{L_{j}^{\prime}}\left\{\begin{array}{l}
\int_{\tau^{\prime}{ }_{j}} W_{3, j}^{l}\left|\left\langle O^{\prime}{ }_{j}(t)\right\rangle\right|^{2} d t, \text { if }{\tau^{\prime}}_{j}^{l} \text { is an interval } \\
W_{3, j}^{l}\left|\left\langle O_{j}^{\prime}\left({\tau^{\prime}}_{j}^{l}\right)\right\rangle\right|^{2}, \text { if }{\tau_{j}^{\prime}}_{j}^{l} \text { is a discrete time }
\end{array}\right.
$$


In addition to those explicitly given here, there are many other forms of $J_{k}$ that could be incorporated into the cost functional. These terms could represent, for example, restrictions on the windowed Fourier transform of $\epsilon(t)$ to a particular frequency band, minimization of sensitivity to small perturbations in the control law (as will be discussed below), or other characteristics of the desired optimal control solution.

One property of control law solutions $C(t)$ of practical import is simplicity. Several measures of simplicity could be used, such as the ability to decompose the control law into only a small number of spectral components with high accuracy (see Geremia et al. (2000)). However, the notion of field simplicity is best associated with the ease of stable and reliable generation in the laboratory, rather than any preconceived sense of simplicity associated with the presence of few field components. Design of simple control laws might be accomplished by introducing a term in the cost functional that favors solutions with suitable characteristics, or in a very ad hoc fashion by starting an iterative optimization algorithm with a simple control field and halting the process while some of this simplicity is still preserved but likely before complete convergence to the control objectives has been achieved. The latter suggestion follows from the observation that the final small fraction of progress toward the control objectives is often responsible for most of the complexity in the control field (e.g. see Table 6.1 of Rice and Zhao (2000) or Tersigni et al. (1990)). None of the above approaches has been subjected to a careful mathematical analysis, and further efforts to characterize the effects of these modifications on the optimal control process may be useful.

Thus far the terms in the cost functional have all been introduced to seek a control field that biases the objective or other goals in some specified direction. Under favorable circumstances one or more of these costs could be re-expressed as a hard demand by introducing a Lagrange multiplier. An example would be a requirement that the laser pulse energy be fixed at a specified laboratory accessible value; the reshaping would redistribute that energy as best as possible over a band of frequency components to meet the physical objective. Some absolute demands may lead to inconsistencies and resultant numerical design difficulties if the demand cannot be satisfied for some (often hidden) dynamical reason.

Once the cost functional has been defined, then the optimal control law is determined by minimizing the cost functional over the function space of admissible controls. Local or global, deterministic or stochastic optimization algorithms (e.g., gradient descent, genetic algorithms, etc.) may be used to find the minimum of the cost functional subject to satisfaction of the Schrödinger equation, possibly under suitable quantum dynamics approximations and assumptions (see Krause and Schafer. (1999); Krause et al. (1998)). The existence of the minimum itself has been investigated in several works, including 
Peirce et al. (1988); for a different formulation see Cancès et al. (2000). Alternatively, the Euler-Lagrange approach may be pursued, as explained below.

At the relevant minima of the cost functional, the first order variation with respect to the control law vanishes:

$$
\frac{\delta J}{\delta C(t)}=0 .
$$

Equation (13.4) is subject to the dynamical constraint that $\psi(t)$ satisfies the Schrödinger equation; this may be assured through the introduction of a Lagrange multiplier function $\lambda(t)$ (cf. Sontag (1998)). The resultant variational problem produces Euler-Lagrange equations whose solutions define the controls $C(t)$ operative at each local extrema of $J$. To demonstrate some of the characteristics of these equations, consider as an example a quantum optimal control problem in which there is only one objective operator $\mathrm{O}$ whose expectation value is to be optimized at the (single target) time $\mathrm{T}$. The control law is taken to be $C(t)=-\mu \epsilon(t)$ and the cost functional $J=J_{1}+J_{2}$ consists of the terms given in Eqns. (13.1) and (13.2) with weights set such that $J$ is given by the Eqn. (5.1). The extended cost functional that includes the Lagrange multiplier $\lambda$, which will be called from now on the adjoint state, is

$$
\begin{array}{r}
J(\epsilon, \psi, \lambda)=<\psi(T)|O| \psi(T)>-\alpha \int_{0}^{T} \epsilon^{2}(t) d t \\
-2 \operatorname{Re}\left[\int_{0}^{T}\left\langle\lambda(t)\left|\frac{\partial}{\partial t}+i\left[H_{0}-\mu \epsilon(t)\right]\right| \psi(t)\right\rangle d t\right]
\end{array}
$$

With this definition we obtain first

$$
\frac{\delta J(\epsilon, \psi, \lambda)}{\delta \epsilon}=-2 \alpha \epsilon(t)-2 \operatorname{Im}\{\langle\lambda(t)|\mu| \psi(t)\rangle\}
$$

The derivative of $J$ with respect to the adjoint state yields (as expected) the equation of motion for the wavefunction. In order to compute the derivative of $J$ with respect to $\psi(t)$ one integrates by parts in the last term of $J(\epsilon, \psi, \lambda)$ and identifies the resulting terms. The Euler-Lagrange equations then become:

$$
\begin{aligned}
& \left\{\begin{array}{l}
i \frac{\partial \psi(t)}{\partial t}=\left[H_{0}-\mu \epsilon(t)\right] \psi(t) \\
\psi(0)=\psi_{0}
\end{array}\right. \\
& \left\{\begin{array}{l}
i \frac{\partial \lambda(t)}{\partial t}=\left[H_{0}-\mu \epsilon(t)\right] \lambda(t) \\
\lambda(T)=O \psi(T)
\end{array}\right. \\
& \alpha \epsilon(t)=-\operatorname{Im}\{\langle\lambda(t)|\mu| \psi(t)\rangle\} .
\end{aligned}
$$


If the field in Eqn. (13.9) is substituted into Eqns. (13.7) and (13.8), the system becomes a pair of coupled nonlinear evolution equations.

Remark 26 Under a mild set of assumptions the general quantum optimal control problem has been shown to possess a countable infinity of solutions, cf. Demiralp and Rabitz (1993). This result has been obtained for cost functionals of the form $J=J_{1}+J_{2}+J_{3}$ having one objective operator $O$ at a final time $T$ and a single penalty operator $O^{\prime}$ evaluated over the entire control interval. In this work additional assumptions are that: i) $O$ and $O^{\prime}$ are bounded operators, ii) $O$ is either positive- or negative-definite, and iii) $\mu \epsilon(t: t \in[0, T])$ is bounded (although the proof can be extended for unbounded control terms).

\section{Numerical algorithms}

This section considers algorithms for determining the optimal controls based on the constrained variational problem in Eqn. (13.4). Part of the text is drawn from the recent review Zhu and Rabitz (in press).

We will consider the quantum optimal control problem with the cost functional given in Eqn. (5.1) and critical point equations (13.7-13.9). The observable operator $O$ is assumed to be positive semidefinite Hermitian. Note that Eqn. (13.9) can also be written as

$$
\begin{aligned}
\epsilon(t) & =\frac{1}{\alpha} \operatorname{Re}\langle\psi(T,\{\epsilon\})|O| \delta \psi(T,\{\epsilon\}) / \delta \epsilon(t)\rangle \\
& =-\frac{1}{\alpha} \operatorname{Im}\langle\psi(T,\{\epsilon\})|O U(T, t,\{\epsilon\}) \mu| \psi(t,\{\epsilon\})\rangle
\end{aligned}
$$

with $U$ being the evolution operator of the system. The presence of the argument $\epsilon$ on the right hand side of Eqn. (14.1) indicates that this is actually an implicit relation for determining the control field. To solve for the control field in Eqn. (13.9) or (14.1), it is evident that some type of iteration algorithm needs to be employed.

Several numerical algorithms can be used; historically, the gradient-type methods (see Shi et al. (1988); Combariza et al. (1991)) were the first to be used. In this approach,

(1) an initial guess $\epsilon_{1}$ is set and the iteration count $k$ is initialized to $k=1$;

(2) the wavefunction $\psi_{k}(t)$ is propagated forward in time with the field $\epsilon_{k}$ by the Eqn. (13.7);

(3) the final data for the adjoint state $\lambda_{k}(T)$ is derived; 
(4) the wavefunction $\psi_{k}(t)$ and the adjoint state $\lambda_{k}(t)$ are propagated backward in time with the field $\epsilon_{k}$ and a new electric field $\epsilon_{k+1}=\epsilon_{k}+\gamma \cdot \frac{\delta J}{\delta \epsilon_{k}}$ is computed $\left(\frac{\delta J}{\delta \epsilon_{k}}\right.$ is given by the Eqn. (13.6)). The constant $\gamma$ is found by a linear search to optimize $J$ in the direction of the gradient $\frac{\delta J}{\delta \epsilon_{k}}$;

(5) $k \leftarrow k+1$; step 2 is returned to and the cycle is continued until convergence.

Note that in step 4 the propagation of the wavefunction $\psi_{k}(t)$ is required because the storage of its trajectory (already computed in step 2) is usually too expensive. We refer the reader to Tersigni et al. (1990) for a conjugated gradient version of the above algorithm.

Although this algorithm proved useful in some cases, its convergence is not guaranteed (here the setting is far from the quadratic cost functional that conjugated gradient type algorithms best optimize) and it may become slow; the algorithm used by Tannor (see Tannor et al. (1992); Somlói et al. (1993)) based on the Krotov method (Krotov $(1973,1974 a, b)$ ) was designed to correct this feature. The structure of this algorithm is as follows:

(1) an initial guess $\epsilon_{1}$ is set and the iteration count $k$ is initialized to $k=1$;

(2) the wavefunction $\psi_{1}(t)$ is propagated with the field $\epsilon_{1}$ by the Eqn. (13.7);

(3) the final data for the adjoint state $\lambda_{k}(T)$ is derived;

(4) the adjoint state $\lambda_{k}(t)$ is propagated backward in time with the field $\epsilon_{k}$ to give $\lambda_{k 0}=\lambda_{k}(0)$

(5) A new field is constructed by the simultaneous resolution of the following equations:

$$
\begin{aligned}
& \left\{\begin{array}{l}
i \frac{\partial \psi_{k+1}(t)}{\partial t}=\left[H_{0}+\frac{1}{\alpha} \mu \operatorname{Im}\left\{\left\langle\lambda_{k}(t)|\mu| \psi_{k+1}(t)\right\rangle\right\}\right] \psi_{k+1}(t) \\
\psi_{k+1}(0)=\psi_{0}
\end{array}\right. \\
& \left\{\begin{array}{l}
i \frac{\partial \lambda_{k}(t)}{\partial t}=\left[H_{0}-\mu \epsilon_{k}(t)\right] \lambda_{k}(t) \\
\lambda_{k}(0)=\lambda_{k 0}
\end{array}\right. \\
& \alpha \epsilon_{k+1}(t)=-\operatorname{Im}\left\{\left\langle\lambda_{k}(t)|\mu| \psi_{k+1}(t)\right\rangle\right\}
\end{aligned}
$$

(6) $k \leftarrow k+1$; step 3 is returned to and the cycle is continued until convergence.

As in step 4 of the gradient descent algorithm, the propagation in Eqn. (14.3) is motivated by memory storage considerations.

In order to analyze the numerical properties of this algorithm we evaluate the difference between the value of the cost functional between two succesive iterations: 


$$
\begin{aligned}
& J\left(\epsilon_{k+1}\right)-J\left(\epsilon_{k}\right)=\left\langle\psi_{k+1}(T)|O| \psi_{k+1}(T)\right\rangle-\alpha \int_{0}^{T} \epsilon_{k+1}(t)^{2} d t \\
& -\left\langle\psi_{k}(T)|O| \psi_{k}(T)\right\rangle+\alpha \int_{0}^{T} \epsilon_{k}(t)^{2} d t \\
& =\left\langle\psi_{k+1}(T)-\psi_{k}(T)|O| \psi_{k+1}(T)-\psi_{k}(T)\right\rangle+2 \operatorname{Re}\left\langle\psi_{k+1}(T)-\psi_{k}(T)|O| \psi_{k}(T)\right\rangle \\
& +\alpha \int_{0}^{T}\left(\epsilon_{k+1}-\epsilon_{k}\right)(t)^{2} d t+2 \alpha \int_{0}^{T}\left(\epsilon_{k}-\epsilon_{k+1}\right)(t) \epsilon_{k+1}(t) d t
\end{aligned}
$$

Since we have also:

$$
\begin{aligned}
& 2 \operatorname{Re}\left\langle\psi_{k+1}(T)-\psi_{k}(T)|O| \psi_{k}(T)\right\rangle=2 \operatorname{Re}\left\langle\psi_{k+1}(T)-\psi_{k}(T), O \psi_{k}(T)\right\rangle \\
& =2 \operatorname{Re}\left\langle\psi_{k+1}(T)-\psi_{k}(T), \lambda_{k}(T)\right\rangle=2 \operatorname{Re} \int_{0}^{T}\left\langle\frac{\partial\left(\psi_{k+1}(t)-\psi_{k}(t)\right)}{\partial t}, \lambda_{k}(t)\right\rangle+ \\
& \left\langle\psi_{k+1}(t)-\psi_{k}(t), \frac{\partial \lambda_{k}(t)}{\partial t}\right\rangle d t= \\
& 2 \operatorname{Re} \int_{0}^{T}\left\langle\frac{H_{0}-\mu \epsilon_{k+1}}{i} \psi_{k+1}(t)-\frac{H_{0}-\mu \epsilon_{k}}{i} \psi_{k}(t), \lambda_{k}(t)\right\rangle \\
& +\left\langle\psi_{k+1}(t)-\psi_{k}(t), \frac{H_{0}-\mu \epsilon_{k}}{i} \lambda_{k}(t)\right\rangle=2 \operatorname{Re} \int_{0}^{T} \epsilon_{k+1}\left\langle\frac{-\mu}{i} \psi_{k+1}(t), \lambda_{k}(t)\right\rangle \\
& -\epsilon_{k}\left\langle\frac{-\mu}{i} \psi_{k}(t), \lambda_{k}(t)\right\rangle+\epsilon_{k}\left\langle\psi_{k+1}(t)-\psi_{k}(t), \frac{-\mu}{i} \lambda_{k}(t)\right\rangle \\
& =2 \int_{0}^{T} \epsilon_{k+1} \cdot \alpha \epsilon_{k+1}-\epsilon_{k} \cdot\left\langle\frac{-\mu}{i} \psi_{k}(t), \lambda_{k}(t)\right\rangle-\epsilon_{k} \cdot \alpha \epsilon_{k+1} \\
& -\epsilon_{k} \cdot\left\langle\psi_{k}(t), \frac{-\mu}{i} \lambda_{k}(t)\right\rangle=2 \alpha \int_{0}^{T} \epsilon_{k+1}(t) \cdot\left(\epsilon_{k+1}-\epsilon_{k}\right)(t) d t
\end{aligned}
$$

we obtain thus from (14.5) and (14.6)

$$
\begin{aligned}
& J\left(\epsilon_{k+1}\right)-J\left(\epsilon_{k}\right)=\left\langle\psi_{k+1}(T)-\psi_{k}(T)|O| \psi_{k+1}(T)-\psi_{k}(T)\right\rangle+ \\
& \alpha \int_{0}^{T}\left(\epsilon_{k+1}-\epsilon_{k}\right)(t)^{2} d t \geq 0
\end{aligned}
$$

because the observable $O$ is a positive semidefinite operator. Each step of this algorithm will therefore result in an increase of the value of the cost functional; this increase is expected to be important for initial steps where the critical point equations are not fulfilled and the difference between succesive fields $\epsilon_{k}$ and $\epsilon_{k+1}$ will be important.

This algorithm was improved with the introduction of the monotonic quadratically convergent algorithm in Zhu et al. (1998); Zhu and Rabitz (1998) that incorporates additional feedback from the adjoint state. The following material will present some aspects of the monotonically convergent algorithms and will analyze their convergence features. In the first step, a trial field $\epsilon^{(0)}(t)$ is used to calculate the wave function $\psi\left(t,\left\{\epsilon^{(0)}\right\}\right)$, then Eqn. (14.1) can be 
directly applied to obtain the first iteration to the control field $\epsilon^{(1)}(t)$ by backward propagation of the evolution operator $U\left(T, t,\left\{\epsilon^{(1)}\right\}\right)$ from $T$ to 0 . In the second step, the new control field $\epsilon^{(2)}(t)$ also can be directly obtained by forward propagation of the wave function $\psi\left(t,\left\{\epsilon^{(2)}\right\}\right)$ from 0 to $T$. A series of control fields can be repeatedly mapped out in this way. The algorithm as an iteration sequence has the following structure:

$$
\begin{gathered}
\epsilon^{(1)}(t)=-\frac{1}{\alpha} \operatorname{Im}\left\langle\psi\left(T,\left\{\epsilon^{(0)}\right\}\right)\left|O U\left(T, t,\left\{\epsilon^{(1)}\right\}\right) \mu\right| \psi\left(t,\left\{\epsilon^{(0)}\right\}\right)\right\rangle \\
\epsilon^{(2)}(t)=-\frac{1}{\alpha} \operatorname{Im}\left\langle\psi\left(T,\left\{\epsilon^{(0)}\right\}\right)\left|O U\left(T, t,\left\{\epsilon^{(1)}\right\}\right) \mu\right| \psi\left(t,\left\{\epsilon^{(2)}\right\}\right)\right\rangle \\
\vdots \\
\epsilon^{(2 i+1)}(t)=-\frac{1}{\alpha} \operatorname{Im}\left\langle\psi\left(T,\left\{\epsilon^{(2 i)}\right\}\right)\left|O U\left(T, t,\left\{\epsilon^{(2 i+1)}\right\}\right) \mu\right| \psi\left(t,\left\{\epsilon^{(2 i)}\right\}\right)\right\rangle(14 . \\
\epsilon^{(2 i+2)}(t)=-\frac{1}{\alpha} \operatorname{Im}\left\langle\psi\left(T,\left\{\epsilon^{(2 i)}\right\}\right)\left|O U\left(T, t,\left\{\epsilon^{(2 i+1)}\right\}\right) \mu\right| \psi\left(t,\left\{\epsilon^{(2 i+2)}\right\}\right) \mathbb{1}\right\} .
\end{gathered}
$$

The introduction of the adjoint state $\lambda$ allows for rewriting the above algorithm in the simplified form

(1) an initial guess $\epsilon_{1}$ is set and the iteration count $k$ is initialized to $k=1$;

(2) the wavefunction $\psi_{0}(t)$ is propagated with the field $\epsilon_{1}$ by Eqn. (13.7);

(3) the final data for the adjoint state $\lambda_{k}(T)$ is derived;

(4) the adjoint state $\lambda_{k}(t)$ is propagated backward in time

$$
\left\{\begin{array}{l}
i \frac{\partial \lambda_{k}(t)}{\partial t}=\left[H_{0}+\frac{1}{\alpha} \mu \operatorname{Im}\left\langle\lambda_{k}(t)|\mu| \psi_{k-1}(t)\right\rangle\right] \lambda_{k}(t) \\
\lambda_{k}(T)=O \psi_{k-1}(T)
\end{array}\right.
$$

Note: if the trajectory of the wavefunction $\psi_{k-1}(t)$ cannot be stored into memory, it is recomputed from the (stored) corresponding field.

(5) the wavefunction is evolved and a new field is constructed by the solution of the following equations:

$$
\begin{aligned}
& \left\{\begin{array}{l}
\left.i \frac{\partial \psi_{k}(t)}{\partial t}=\left[H_{0}+\frac{1}{\alpha} \mu \operatorname{Im}\left\langle\lambda_{k}(t)|\mu| \psi_{k}(t)\right\rangle\right]\right] \psi_{k}(t) \\
\psi_{k}(0)=\psi_{0}
\end{array}\right. \\
& \alpha \epsilon_{k+1}(t)=-\operatorname{Im}\left\{\left\langle\lambda_{k}(t)|\mu| \psi_{k}(t)\right\rangle\right\}
\end{aligned}
$$

As in step 4, if needed, the trajectory of the adjoint state $\lambda_{k}(t)$ is recomputed.

(6) $k \leftarrow k+1$; step 3 is returned to and the cycle is contined until convergence.

In order to analyze the convergence features of the above iteration sequence, we go back to the formulation in Eqns. (14.10) and (14.11) and consider the 
deviation of the objective functional between two neighboring steps (one step is defined as a pair of backward and forward propagations). Specifically, between the $i$-th and $(i+1)$-st steps, the deviation is

$$
\begin{aligned}
\Delta J_{i+1, i}= & \left\{\left\langle\psi\left(T,\left\{\epsilon^{(2 i+2)}\right\}\right)|O| \psi\left(T,\left\{\epsilon^{(2 i+2)}\right\}\right)\right\rangle-\alpha \int_{0}^{T}\left[\epsilon^{(2 i+2)}(t)\right]^{2} d t\right\} \\
& -\left\{\left\langle\psi\left(T,\left\{\epsilon^{(2 i)}\right\}\right)|O| \psi\left(T,\left\{\epsilon^{(2 i)}\right\}\right)\right\rangle-\alpha \int_{0}^{T}\left[\epsilon^{(2 i)}(t)\right]^{2} d t\right\}
\end{aligned}
$$

Considering the dynamical equations of the wave functions $\psi\left(t,\left\{\epsilon^{(2 i+2)}\right\}\right)$ and $\psi\left(t,\left\{\epsilon^{(2 i)}\right\}\right)$, and utilizing the field expressions in Eqns. (14.10) and (14.11), it can be derived (see Zhu and Rabitz (1998)) that:

$$
\begin{aligned}
\Delta J_{i+1, i}= & \alpha \int_{0}^{T}\left(\left[\epsilon^{(2 i+2)}(t)-\epsilon^{(2 i+1)}(t)\right]^{2}+\left[\epsilon^{(2 i+1)}(t)-\epsilon^{(2 i)}(t)\right]^{2}\right) d t \\
& +\left\langle\Delta \psi_{i+1, i}(T)|O| \Delta \psi_{i+1, i}(T)\right\rangle
\end{aligned}
$$

where $\Delta \psi_{i+1, i}(t) \equiv \psi\left(t,\left\{\epsilon^{(2 i+2)}\right\}\right)-\psi\left(t,\left\{\epsilon^{(2 i)}\right\}\right)$. Since $O$ is a positive semidefinite operator,

$$
\left\langle\Delta \psi_{i+1, i}(T)|O| \Delta \psi_{i+1, i}(T)\right\rangle \geq 0,
$$

then Eqn. (14.16) satisfies

$$
\Delta J_{i+1, i} \geq 0
$$

where the equal sign occurs only if the initial guess for the control field happens to be an exact solution of Eqn. (14.1). Equation (14.18) indicates that regardless of the choice for the initial trial field, the objective functional will monotonically converge to a local maximum of $J$ for the iterated sequence of control fields given in Eqns. (14.8-14.11).

It is straightforward to show that the total gain for the objective functional after $N$ iteration steps will be

$$
\Delta J_{N, 0}=\sum_{i=0}^{N-1}\left\langle\Delta \psi_{i+1, i}(T)|O| \Delta \psi_{i+1, i}(T)\right\rangle+\alpha \int_{0}^{T} \sum_{i=0}^{2 N-1}\left[\Delta \epsilon_{i+1, i}(t)\right]^{2} d t
$$

where $\Delta \epsilon_{i+1, i}(t) \equiv \epsilon^{(i+1)}(t)-\epsilon^{(i)}(t)$. Based on the above analysis, it can be concluded that a larger change of the field between neighboring steps will lead to faster convergence. As the initial guessed field usually will be far from the exact solution, the major contribution to the rapid convergence is expected to come from the first few iteration steps. An illustration of the monotonic convergence is shown in Figure 3. 
It is worthwhile to point out that the positive semidefiniteness of the operator $O$ is not an intrinsic constraint on the monotonically convergent iteration algorithms: other types of monotonically convergent algorithms can lift this constraint. In the following, we will show one such procedure which is still monotonically convergent, but without the need to impose the positive semidefiniteness constraint on the operator. In the revised algorithm, the iteration sequence for the control field is slightly different from that shown in Eqns. (14.8-14.11). First, an initial guess for the field $\epsilon^{(0)}(t)$ is needed to forward propagate the wave function $\psi\left(t,\left\{\epsilon^{(0)}\right\}\right)$. The modification of the algorithm just lies in the backward propagation. Specifically, the iteration sequence for determining the control field is (Zhu and Rabitz (1999a))

$$
\begin{aligned}
& \left.\epsilon^{(1)}(t)=-\frac{1}{\alpha} \operatorname{Im}\left\langle\psi\left(t,\left\{\epsilon^{(0)}\right\}\right)\left|U^{\dagger}\left(T, t,\left\{\epsilon^{(1)}\right\}\right) O U\left(T, t,\left\{\epsilon^{(1)}\right\}\right) \mu\right| \psi\left(t,\left\{\epsilon^{(0)}\right\}\right\}\right\rangle\right) \\
& \epsilon^{(2)}(t)=-\frac{1}{\alpha} \operatorname{Im}\left\langle\psi\left(t,\left\{\epsilon^{(2)}\right\}\right)\left|U^{\dagger}\left(T, t,\left\{\epsilon^{(1)}\right\}\right) O U\left(T, t,\left\{\epsilon^{(1)}\right\}\right) \mu\right| \psi\left(t,\left\{\epsilon^{(2)}\right\}\right\}\right\rangle 4 \\
& \vdots \\
& \epsilon^{(2 i+1)}(t)=-\frac{1}{\alpha} \operatorname{Im}\left\langle\psi\left(t,\left\{\epsilon^{(2 i)}\right\}\right)\right| U^{\dagger}\left(T, t,\left\{\epsilon^{(2 i+1)}\right\}\right) O U\left(T, t,\left\{\epsilon^{(2 i+1)}\right\}\right) \mu \\
& \left|\psi\left(t,\left\{\epsilon^{(2 i)}\right\}\right)\right\rangle \\
& \epsilon^{(2 i+2)}(t)=-\frac{1}{\alpha} \operatorname{Im}\left\langle\psi\left(t,\left\{\epsilon^{(2 i+2)}\right\}\right)\right| U^{\dagger}\left(T, t,\left\{\epsilon^{(2 i+1)}\right\}\right) O U\left(T, t,\left\{\epsilon^{(2 i+1)}\right\}\right) \mu \\
& \left|\psi\left(t,\left\{\epsilon^{(2 i+2)}\right\}\right)\right\rangle
\end{aligned}
$$

Remark 27 This second algorithm does not lend itself to an implementation in terms of direct and adjoint state only. The propagation of the wavefunction $\psi(t)$ and of the evolution operator $U(T, t,\{\epsilon\})$ that is required is more costly than the propagation of $\psi(t)$ and $\lambda(t)$ of the previous monotonic convergent algorithm version because, in discrete form, it involves propagating a matrix and a vector as opposed to propagating two vectors.

To verify the monotonically convergent feature of the above algorithm, once again we evaluate the deviation of the objective functional between two neighboring steps as follows:

$$
\begin{aligned}
\Delta J_{i+1, i}= & \left\{\left\langle\psi\left(T,\left\{\epsilon^{(2 i+2)}\right\}\right)|O| \psi\left(T,\left\{\epsilon^{(2 i+2)}\right\}\right)\right\rangle-\alpha \int_{0}^{T}\left[\epsilon^{(2 i+2)}(t)\right]^{2} d t\right\} \\
& -\left\{\left\langle\psi\left(T,\left\{\epsilon^{(2 i)}\right\}\right)|O| \psi\left(T,\left\{\epsilon^{(2 i)}\right\}\right)\right\rangle-\alpha \int_{0}^{T}\left[\epsilon^{(2 i)}(t)\right]^{2} d t\right\}(14 .
\end{aligned}
$$

Considering the dynamical equations of the wave function $\psi\left(t,\left\{\epsilon^{(2 i+2)}\right\}\right)$ and 
the operator $U\left(T, t,\left\{\epsilon^{(2 i+3)}\right\}\right)$, and utilizing the field expressions in Eqns. (14.22) and (14.23), it can be proven (e.g. Zhu and Rabitz (1999a)) that:

$$
\Delta J_{i+1, i}=\alpha \int_{0}^{T}\left(\left[\epsilon^{(2 i+2)}(t)-\epsilon^{(2 i+1)}(t)\right]^{2}+\left[\epsilon^{(2 i+1)}(t)-\epsilon^{(2 i)}(t)\right]^{2}\right) d t(.14 .25)
$$

Comparing Eqn. (14.25) with Eqn. (14.16), we see that the iteration algorithm given by Eqns. (14.20-14.23) is still monotonically convergent for the objective functional, but without the extra constraint on the operator $O$. The total monotonic gain of the objective functional after $N$ iteration steps is simply

$$
\Delta J_{N, 0}=\alpha \int_{0}^{T} \sum_{i=0}^{2 N-1}\left[\Delta \epsilon_{i+1, i}(t)\right]^{2} d t
$$

We will not go further here to explore other possible monotonically convergent algorithms to iteratively solve for optimal controls. It is important to keep in mind that monotonically convergent iteration algorithms may not exist for arbitrary objective functionals. However, extended work indicates that there exist monotonically convergent iteration algorithms for most common types of the objective functionals considered in the optimal control of quantum systems Zhu et al. (1998); Schirmer et al. (2000), and that methods similar to those presented here may be applied to the density matrix formulation (Ohtsuki et al. (1999)).

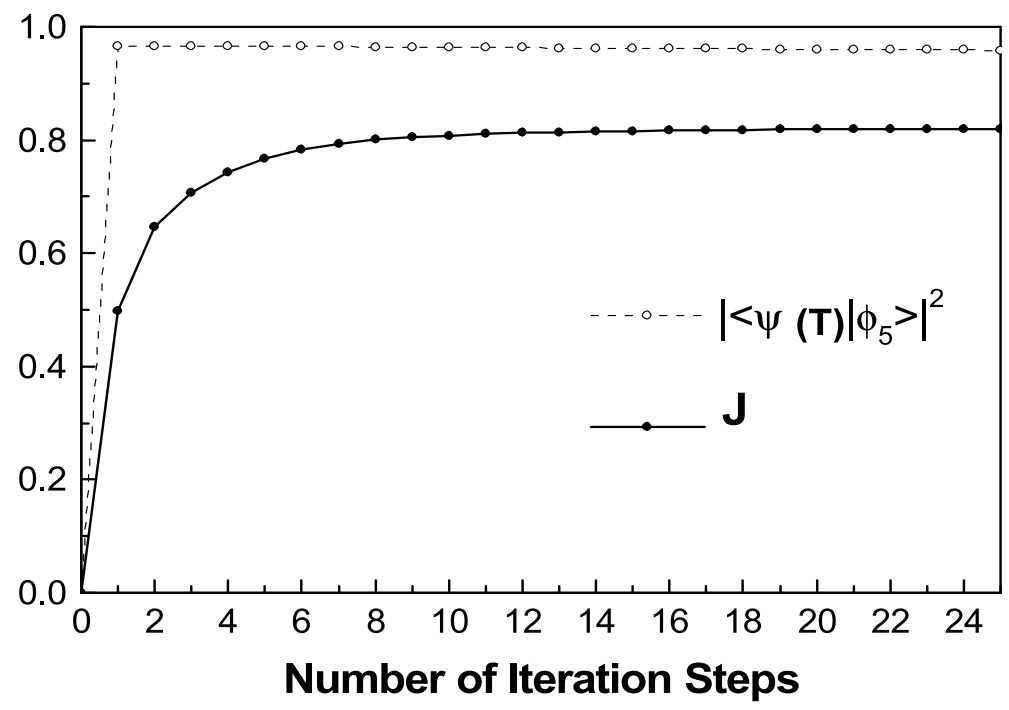

Fig. 3. Convergence of the cost functional to a (locally) optimal value in a numerical implementation of the quadratically convergent algorithm discussed in Section 14. The details of the calculation, which designs a minimum-fluence laser field to promote the $|3\rangle \rightarrow|5\rangle$ vibrational transition in an $\mathrm{O}-\mathrm{H}$ bond, are given in Zhu and Rabitz (in press).

Remark 28 Besides these families of algorithms that aim to solve the critical 
point equations directly, an alternative approach has been proposed in Shen et al. (1994) that uses a penalization framework. Suppose that the objective can be expressed as $\langle\psi(T)|P| \psi(T)\rangle=1$, where $P$ is a projection operator; $a$ sequence of cost functionals

$$
J(\epsilon, \gamma)=\int_{0}^{T} \epsilon^{2}(t) d t+\frac{1}{2 \gamma}\|\langle\psi(T)|P| \psi(T)\rangle-1\|^{2}
$$

(where $\psi(t)$ is the solution of Eqn. (13.7)) is optimized with respect to $\epsilon$ for $\gamma \rightarrow 0$ by an inexact Newton method. The sequence of solutions of these optimization problems converges then to the solution of the initial control problem.

Remark 29 The numerical resolution of the evolution equations as in Eqns. (13.7), (13.8),(14.2),(14.3),(14.12) or (14.13) requires a propagation scheme. Often used is the split-operator technique (e.g. Zhu and Rabitz (1998)) which can be written schematically: suppose that the equation to be solved is

$$
i \frac{\partial}{\partial t} \chi(t)=(K+V(t)) \chi(t)
$$

where $K$ is the kinetic energy operator and $V(t)$ is the total potential. Then, denoting by $\Delta t$ the time step, the following recurrence is used:

$$
\chi(t+\Delta t)=e^{-i K \Delta t / 2} e^{-i V(t) \Delta t} e^{-i K \Delta t / 2} \chi(t)
$$

which is known to be exact to second order in $\Delta t$. In order to apply the operators $e^{-i K \Delta t / 2}$ and $e^{-i V(t) \Delta t}$ a dual real space $\leftrightarrow$ Fourier(momentum) representation is used; note that $V(t)$ is diagonal in real space while $K$ is diagonal in momentum space; each operator is thus applied efficiently, with the transformation from one representation to the other realized by (fast) Fourier transforms.

\section{Robust designs}

Due to imperfect knowledge of system Hamiltonians and coupling operators as well as the limited precision and presence of background fluctuations inherent to any laboratory apparatus, it is impossible to perfectly reproduce either optimally designed control laws or the exact specifications under which they were designed. Hence, it is important to study the sensitivity of the control objective or cost functional to random variations or uncertainties in the operators and initial conditions describing the evolution of the system. There is extensive work on the general topic of robust optimal control in the engineering (Dorato (1987); Hosoe (1991); Ackerman (1985)) and quantum control 
literatures (Demiralp and Rabitz (1998); Beumee et al. (1990); Zhang and Rabitz (1994)).

A general approach to assessing robustness and stability in quantum control has been considered in Demiralp and Rabitz (1998) based on introducing a stability operator $S$, the kernel of which is related to the curvature $\frac{\delta^{2} J[\epsilon]}{\delta \epsilon(t) \delta \epsilon\left(t^{\prime}\right)}$ of the cost functional with respect to the control law. Considering the curvature is necessary as the null value of the first order variation $\frac{\delta J[\epsilon]}{\delta \epsilon(t)}=0$ defines the optimal solution. Conditions for robustness and optimality of the control solutions can be expressed in terms of the spectrum of $S$, and this analysis can also reveal qualitative relationships between the various terms in the cost functional and the robustness/optimality features of the control solutions. Work is still needed in order to find a general relationship between the dominant characteristics of a system Hamiltonian, coupling operators, and the cost functional with respect to the eigenvalues of the stability operator $S$.

The introduction of a penalty term of the form

$$
J_{3}=\int_{0}^{T} W_{3}\left|\left\langle\psi(t)\left|O^{\prime}\right| \psi(t)\right\rangle\right|^{2} d t
$$

where $O^{\prime}$ is an arbitrary positive definite operator, was observed (Demiralp and Rabitz (1998)) to improve the robustness of optimal control solutions. The presence of $J_{3}$ can bias the system to satisfy demands tangential to the true control objectives, causing an effective "drag" along the way to the goal. Hence, the effect of $J_{3}$ may be loosely interpreted as analogous to the presence of viscous drag in stabilizing a classical mechanical system about a weakly stable point in its phase space. However, the possible stabilization mechanisms have not been carefully studied or characterized and a complete mechanism to explain how the introduction of suitable ancillary objectives may stabilize the solutions to quantum optimal control problems remains to be found.

The robustness effects of penalty operators with more specific forms than that given by Eqn. (15.1) may be easier to intuit. For example, the term

$$
J_{s}=\int_{0}^{T} d t\left(\frac{\delta\langle O(T)\rangle}{\delta \epsilon(t)}\right)^{2}
$$

(or analogous expressions with higher derivatives) may be used (Kobayashi (1998)) to reduce the sensitivity of the achieved control objectives at the target time $T$ to uncertainty in control fields. Analogs of this penalty term for the sensitivity of the target objective to uncertainty in other variables were found (see Beumee et al. (1990)) to be capable of reducing the sensitivity to errors in force constants and other model parameters.

Design of robust quantum optimal control solutions can be achieved through 
the min-max procedure, which involves simultaneously maximizing the effects of an energy-bounded disturbance and minimizing the objective functional. Solutions to such min-max problems represent the best possible control under the worst possible energy-bounded disturbances. For linear dynamical systems the min-max problem becomes $H_{\infty}$ control, which has an exact solution through the Ricatti equations. This procedure is well-developed in engineering control theory (see e.g. Athans and Falb (1966)), and it has been applied to robust control designs for selective vibrational excitation in molecular harmonic oscillators (Beumee and Rabitz (1992)).

Remark 30 In general, the min-max technique tends to give conservative robust solutions as it works against the worst possible bounded disturbance, and encountering this worst disturbance in practice is unlikely. This point suggests that consideration of a less extreme class of disturbances may also give useful solutions. The resulting analysis should give designs that are robust under more realistic conditions than those modeled in a worst case scenario.

The conclusions of min-max studies (Zhang and Rabitz (1994)) reinforce the importance of this remark. For a diatomic molecule modeled as a Morse oscillator, the robustness properties of solutions to the min-max equations were compared with solutions to the standard Euler-Lagrange equations (c.f. Eqns. (13.7)-(13.9)) derived without any robustness considerations. While the min-max controls performed better under the application of the worst-possible disturbance (for which they were designed), they did not necessarily outperform the standard Euler-Lagrange solutions under disturbances other than the worst case. For example, min-max control fields were demonstrated to be significantly less-robust than standard Euler-Lagrange control fields to sinusoidal perturbations with the same amplitude constraints as the worst-case disturbance. This underscores the importance of designing control laws that are robust to the particular class of disturbances most likely to occur.

Even in cases where the robustness properties of two designs are quite distinct, simulations have shown (see Beumee and Rabitz (1992)) that robust control designs may differ only slightly from nonrobust designs (i.e., the $L^{2}$ norm of the difference between the two control laws may be small). This similarity suggests that robustness properties in some cases may result from very subtle effects. It has also been noted (Beumee and Rabitz (1992)) that the relationship between the robust field and the standard design (i.e., created without robustness considerations) can take two forms: the robust field can be either a scaled, self-similar version of the standard field (which may be described as achieving robustness by "speaking louder") or can have a qualitatively different form. At present, no means exists to predict in general when either of these two cases will occur. It is suggestive that self-similar robust fields will exist for weak disturbances, but there is presently no proof of this conjecture. 


\section{Tracking}

As mentioned in Remark 26 of Chapter 4, there generally exist a multiplicity of solutions to the quantum optimal control equations, suggesting that it may be possible to predefine a selected path between the initial and final conditions satisfying the control objectives. The existence of such a path exactly matching the conditions at both ends assumes that the system is controllable. The path $y(t)$ can be implicitly defined by the expectation value of a tracking operator $O_{t r}$ :

$$
y(t)=\left\langle\psi(t)\left|O_{t r}\right| \psi(t)\right\rangle ; \quad t \in[0, T]
$$

The quantum tracking control problem (e.g. Gross et al. (1993b); Lu and Rabitz (1995); Ohtsuki et al. (1998); Ong et al. (1984)) may be viewed as a special case of optimal control theory with the target being the expectation value of $O_{t r}$ over the entire time interval. (In some cases it may be physically attractive to only require that $\lim _{t \rightarrow T} O_{t r}(t)=O$, where $\mathrm{O}$ is the objective operator whose expectation value is desired at T.) Given the path defined in Eqn. (16.1), the tracking algorithm for determining the control law may be derived from the Heisenberg equation of motion

$$
i \hbar \frac{d\left\langle\psi(t)\left|O_{t r}\right| \psi(t)\right\rangle}{d t}=\left\langle\psi(t)\left|\left[H, O_{t r}\right]\right| \psi(t)\right\rangle+\left\langle\psi(t)\left|\frac{\partial O_{t r}}{\partial t}\right| \psi(t)\right\rangle .
$$

With a control law of the form $C(t)=-\mu \epsilon(t)$ and the assumptions that $O_{t r}$ is independent of time along with

$$
\left[\mu, O_{t r}\right] \neq 0
$$

Eqn. (16.2) can be rewritten to solve for the electric field:

$$
\epsilon(t)=\left(i \hbar \frac{d y}{d t}-\left\langle\psi(t)\left|\left[H_{0}, O_{t r}\right]\right| \psi(t)\right\rangle\right) /\left\langle\psi(t)\left|\left[\mu, O_{t r}\right]\right| \psi(t)\right\rangle .
$$

This equation may be substituted into the Schrödinger equation, which then can be numerically solved for $\psi(t)$; substituting $\psi(t)$ back into Eqn. (16.3) gives an explicit expression for the required control law. One important feature of this technique is that it requires only a single numerical solution of the Schrödinger equation, as opposed to the iterative methods of standard optimal control.

Given the freedom in the selection of $y(t)$, one might unknowingly choose a track that generates one or more singularities, or events at which the denominator of the control field in Eqn. (16.3) vanishes. This type of singularity may be classified as trivial (see Zhu et al. (1999)) if it exists for all $t \in[0, T]$. Trivial 
singularities may be removed by formulating a tracking equation analogous to Eqn. (16.1) for control of the $k$-th time-derivatives of $y(t)$. A rank index may be assigned to each tracking singularity by determining the smallest order $k_{r}$ for which the corresponding tracking equation has no trivial singularity; if the rank index is infinite, then the track-system pair is uncontrollable. Otherwise, any remaining (isolated) singularities may be treated as nontrivial singularities of some relative order $k_{n t}$ (as in Zhu et al. (1999)). The magnitude of the disturbance to the trajectory resulting from a nontrivial singularity depends inversely on the magnitude of the derivatives $\frac{\partial^{i} y}{\partial t^{i}}, i=1, \ldots, k_{n t}$ evaluated at the singularity. This partially explains the effects of singularities on quantum tracking control and encourages the search for a non-iterative algorithm to sense the occurrence of a forthcoming singularity and accordingly alter the path to avoid the momentary singularity while eventually reaching the objective.

Several extensions of exact inverse tracking which relax demands that could otherwise produce physically unreasonable fields have been developed in Chen et al. (1995). The first of these methods is local track generation, in which the problems associated with an a priori trajectory design are avoided by letting the track depend on the evolving quantum state: $y(t)=y(\psi(t))$. This approach is especially useful when the control objectives are not specifically defined by target operator expectation values as in Eqn. (4.1), but rather can be expressed as the production of some qualitative change in a system. A second method is asymptotic tracking, in which the operator $O_{t r}$ is modified to allow an asymptotic approach to possibly singular trajectories. Finally, in the competitive tracking technique a cost functional is defined whose minimization produces a solution optimally matching a number of trajectories for different tracking operators as well as minimizing the field fluence or satisfying other control objectives.

There is considerable room for further development of the tracking procedure guided by the attraction of performing only one solution of the Schrödinger equation to achieve a control design. Moreover, thus far tracking control has only been applied to the wave function formulation of quantum mechanics. A significant extension would be to treat mixed states in the density matrix formulation. In this context, the expectation value $\left\langle O_{t r}(t)\right\rangle=\operatorname{Tr}\left(\rho(t) O_{t r}\right)$ would be followed and the Schrödinger equation would be replaced by Eqn. (7.1), with the possibility of additionally including decoherence processes.

\section{Laboratory achievement of closed loop control}

The design of control laws poses interesting theoretical challenges, and the practical motivation for such a task is to accomplish successful control in 


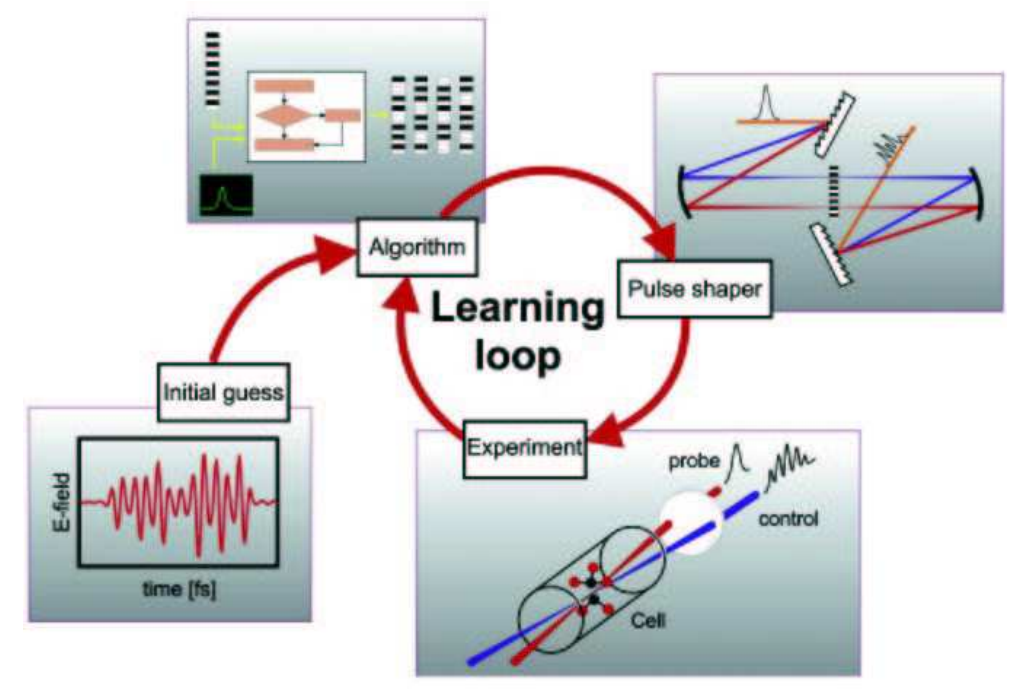

Fig. 4. A closed-loop process for teaching a laser to control quantum systems. The loop is entered with either an initial design estimate of even a random field in some cases. A current laser control field design is created with a pulse shaper and then applied to the sample. The action of the control is assessed, and the results are fed to a learning algorithm to suggest an improved field design for repeated excursions around the loop until the objective is satisfactory achieved. From Rabitz et al. (2000) with permission from the copyright owner.

the laboratory. This section discusses the conceptual and theoretical aspects of laboratory operations in which information about the evolving quantum systems is used to improve or define effective control laws. We will cover the technique of quantum learning control, which is increasingly proving to be the most efficient method of practically achieving many control objectives, especially in complex quantum systems; we will also discuss aspects of feedback quantum control. Learning and feedback control are closed loop experimental procedures aimed at achieving control even in the presence of Hamiltonian uncertainties and laboratory disturbances.

The computational design of a control law to meet a physical objective requires (i) explicit knowledge of the system Hamiltonian and (ii) the ability to numerically solve the quantum control equations at least once (in the case of tracking control) or many times for convergence to an optimal solution. In practice, however, these requirements can rarely be met. If the system to be controlled is sufficiently complex (e.g., a polyatomic molecule), it is likely that the Hamiltonian will be only approximately known and the corresponding quantum design equations can only be solved under serious approximations.

In light of these limitations, a completely different and practical approach to the control of quantum dynamics phenomena has been developed (see Judson and Rabitz (1992)). In this quantum learning control technique, the laboratory quantum system in itself serves as an analog computer to guide its own 
control as indicated in Figure 4. This approach addresses the requirements of (i) and (ii) above: a physical quantum system can solve its Schrödinger equation in real time and with exact knowledge of its own Hamiltonian, all without computational cost to the user. Hence, the burden of knowing the Hamiltonian and solving the Schrödinger equation is shifted over to a laboratory effort with a learning algorithm guiding the control experiments. The number of physi$\mathrm{cal} /$ chemical systems treated in this way is growing rapidly, and in many cases it is easier to do the experiments than to perform the designs. However, this approach can still benefit from even approximate control designs to start the laboratory learning process, and theory also has an important role to play in introducing the proper stable and reliable algorithms to make the experiments successful.

In summarizing the methodology of quantum learning control, we will consider a simple paradigm where the state of the system is to be optimized at the final time $T$ only. The first step is to prepare the laboratory quantum system in a convenient initial state $\psi(0)=\psi_{0}$, or a mixed state or a distribution of incoherent states specified by $\rho(0)$. Next, the system is allowed to evolve under its Hamiltonian and some initial trial control law $C_{0}$ applied in the laboratory. At the time $T$, a measurement of the corresponding control objective(s) is made. The quantum system (perturbed by this measurement) is then discarded, and the control law may be updated to $C_{1}$ based on the information gained through this measurement. The method and the frequency with which the control law is updated depends on the specific learning algorithm being used (e.g., as described below, with a genetic algorithm the control law is updated after some multiple of $N_{\text {pop }}$ experiments in each time interval). This updating continues until the learning algorithm has converged to some final control law $C$ that achieves the objectives within the convergence bounds of the learning algorithm. The methods most widely used to accomplish the updating of learning control laws are evolutionary and genetic algorithms (GAs) (e.g. Goldberg (1989)), although other learning algorithms could be used.

A GA involves the evolution of successive generations of control laws from their parents, in some fashion mimicking biological evolution. Each trial control field is encoded to form a gene which is part of an overall population evolved in the laboratory during the search for an optimal control. Specifically, experiments are performed in which the quantum system evolves under each of the members of a control law "population". The "fitness" of the control law is evaluated based on the degree to which the control objective(s) are achieved, and the fittest control laws are preserved and/or modified in some prescribed fashion in the next generation. This procedure is continued until the fittest members of the control law population achieve the control objectives to the required extent.

Remark 31 A more general learning control setting may include a discrete 
(yet finite) set of objective times in $\left\{t_{i}: i=1, \ldots, n\right\}$ or more generally unions of discrete and continuous time intervals. When more than one target is present the problem is divided into several independent subproblems solved sequentially in time. Note that all the experiments carried out thus far (e.g.,Assion et al. (1998); Bergt et al. (1999); Weinacht et al. (1999); Bardeen et al. (1997a, 1998); Hornung et al. (2000); Levis et al. (2001)) have dealt with a single target goal.

The power of the GA and evolutionary algorithms lies in their ability to globally search the space of control laws and discover solutions that may be highly nonintuitive. This directed search takes advantage of the ability to perform a great number of distinct control experiments in a short period of laboratory time, and the closed loop technique has been demonstrated for a wide variety of quantum systems and control objectives. The method has also been shown to have interesting convergence properties. For example, it is observed in simulations (as in Judson and Rabitz (1992)) and experiments (e.g. Assion et al. (1998); Bergt et al. (1999); Weinacht et al. (1999); Bardeen et al. (1997a, 1998); Levis et al. (2001)) that the GA algorithm can converge for a set of randomly constructed initial control law populations. It is then interesting to know to what extent will the convergence properties of these algorithms be improved by incorporating trial designs into the search space.

Remark 32 The choice of cost functionals (see Section 13) used in the experiments has the same freedom as for computational optimal control theory except that in laboratory learning control there is no direct access to the wave functions. At present the experiments have considered only the final target in the cost, but other criteria could be included giving rise to a possible enhancement to the procedure. Figure 5 shows an application of the laboratory learning control concept.

Simulations have considered the effects of laboratory errors (modeled as distortions, or transformations, of true input and output data) and noise upon the learning control process (Gross et al. (1993a); Tóth et al. (1994)). In Tóth et al. (1994), input errors were modeled by performing various functional transformations on the control laws used in simulated experiments, while output errors were represented by transforming the expectation values of the control objectives corresponding to these experiments. In general, if the input errors are systematic and the output errors are random, they may not significantly affect the ability of the learning algorithm to find an optimal solution. The fitness of the final control laws found by the GA are also demonstrated to be reasonably insensitive to noise in the control fields. These results are based on very limited studies of simple model systems, and they invite further investigation. Such an analysis could give insight into how best to operate the laboratory experiments. 


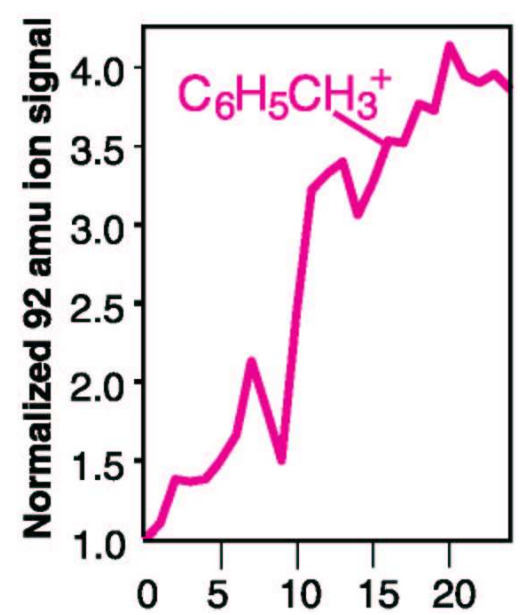

Fig. 5. An example the laboratory closed-loop control process. The objective, to maximize the flux of toluene (ions) from the dissociation rearrangement of acetophenone is achieved as the control law evolves over successive generations, see Levis et al. (2001).

In principle, any optimization algorithm can be applied to quantum learning control. For example, gradient descent and simulated annealing algorithms have been explored in simulations (Gross et al. (1993a)), but the GA outperformed them in several test cases. However, this subject has not received a thorough examination and there may exist algorithms that converge with greater efficiency or robustness than the genetic algorithm for certain classes of quantum mechanical learning control problems. In treating this topic it is important to consider the ability to perform very large numbers of quantum control experiments, which may overcome certain algorithmic shortcomings found under more common conditions. This ability is almost unprecedented in other applications of learning algorithms.

Another approach to quantum learning control is provided by the use of input $\rightarrow$ output mapping techniques as in Phan and Rabitz (1997, 1999); Geremia et al. (2001). These methods develop an effective map between the inputs (i.e., the parameters or features defining the control laws) and the outputs (i.e., the expectation values of objective operators). A map from the control input space $\mathcal{C}$ to the space of possible expectation values may be determined directly from the laboratory input and output data; a series of these maps may be needed to cover a sufficiently large portion of $\mathcal{C}$. The control law that optimally satisfies the objectives can be identified from these maps using a suitable learning algorithm. A central issue is establishing the efficiency of mapping techniques as compared with eliminating the maps altogether in favor of having the learning algorithm directly interfaced with the laboratory experiments. Beyond issues of efficiency, mapping techniques may offer the additional benefit of providing physical insight into control mechanisms based on the observed map structure. 


\section{Chapter 5. Challenges for the future}

Previous sections have presented the general framework of quantum control, the central issue of controllability, the numerical algorithms for designing control laws and the algorithms for their laboratory discovery. Along the way, several unresolved questions and topics of current interest were introduced. The purpose of the current section is to highlight some emerging areas and unresolved questions that were not a part of this overview. Most of the material is drawn from Brown and Rabitz (in press).

\section{Coherence and control}

The robustness of coherences for controlled quantum systems in mixed states (i.e. the robustness to decay of the off-diagonal terms in the density operator (cf. Section 7) is a topic of special interest. The effects associated with this decay are especially important in the quantum information sciences (see Chuang et al. (1995)), where the development of methods to curtail decoherence in information processing algorithms is an active area of research (e.g. Shor (1995); Steane (1998)). A relevant contribution from control might be the combination of min-max optimal control techniques with the ideas of decoherence-free subspaces (Lidar et al. (1998, 1999); Bacon et al. (1999)), in which dynamics are invariant to interference that would otherwise cause coherences to decay (this is related to the general notion of disturbance decoupling in mathematical systems theory, see Sontag (1998)). The result would be control solutions that maximize coherences while simultaneously minimizing an objective cost functional, which could be of significance in developing a physical understanding of the mechanisms of decoherence and its suppression. We note that other schemes are also being considered for the dynamic manipulation of decoherence and control in the presence of dissipation, such as Viola and Lloyd (1998); Vitali and Tombesi (1999); Cao et al. (1997); Duan and Guo (1999).

When the persistence of coherence is not directly important to applications, it was shown in Bardeen et al. (1999) that coherence may not be operative in the control mechanism. In an $n$-dimensional space $\mathcal{H}_{n}$ and in the presence of rapid dephasing (which implies vanishing of the off-diagonal coherence terms of the density matrix), Eqn. (7.1) reduces to a set of rate equations for the population in the $n$ states. A case of special interest in this regard is the control of condensed phases. Under certain conditions, Bardeen et al. (1999) demonstrates that successful controls can be designed for quantum systems whose evolution is determined by these rate equations. This raises the question of whether quantitative measures of coherence can be developed to assess its role in any quantum control problem. 
The application of control methods to the cooling of quantum systems is an active area of research (Bartana and Kosloff (1993, 1997); Tannor and Bartana (1999); Tannor et al. (1999)) and there are several means of defining cooling on the molecular scale. One typically utilized criterion (see Bartana and Kosloff (1997)) aims to minimize the von Neumann entropy $\sigma=-\sum_{k} p_{k} \log p_{k}$ corresponding to some observable $O$ (such as the system Hamiltonian); here, $p_{k}$ is the probability that the system is in the $k$-th eigenstate of $O$. Another system cooling criterion is to increase in the Reyni entropy $\operatorname{Tr}\left(\rho^{2}\right)$ (as in Bartana and Kosloff (1997)). With both measures, maximal cooling is achieved when all but one of the $p_{k}$ are zero (i.e., achievement of a pure state). Thus, the ability to completely cool a molecular system is likely to be a challenging task in the presence of laser noise: for the purposes of molecular cooling, a laser control with noise fluctuations may be thought of as having an effective nonzero "temperature." Lower bounds related to a temperature beyond which a system cannot be cooled, if they exist, remain to be established.

\section{Can noise help attain control ?}

In general, noise in $C(t)$ is thought of as harmful in the context of trying to achieve control objectives. However, hints from the subject of stochastic resonance (Gammaitoni (1998)) suggest that under suitable conditions noise may possibly have beneficial effects, such as allowing the achievement of a particular level of control using smaller total field fluence than that required for the noise-free system. It remains to be demonstrated under what, if any, conditions the presence of noise can assist in the achievement of quantum control objectives, and to elucidate the possible physical mechanisms behind these effects. In addition, the process of seeking the optimal control will attempt to eliminate the deleterious influence of noise while attempting to reach the objectives.

\section{Qualitative behavior of optimal control solutions}

A large body of numerical studies provides examples of solutions to the quantum optimal control equations. However, none of this work has illuminated the general behavior, stability, and classes of solutions to the quantum optimal control equations (here, by classes of solutions we mean the qualitative notion of groups of solutions with particular properties, such as nondispersivity, periodicity, etc.). The possibility that unusual behavior can be expected is evident from one study which showed that the optimal control equations can be made equivalent to the standard non-linear Schrödinger equation under 
suitable conditions (Demiralp and Rabitz (1997)) (see also in Section 21 the considerations on the deterministic feedback control).

\section{Feedback quantum control}

This section deals with the effects of real-time, laboratory measurements of the quantum system under two distinct experimental schemes, each described by a different type of feedback Schrödinger equation. The first scheme uses a sequence of repeated experiments to avoid the disturbance effects of measurement, while the second directly confronts these effects.

\section{Deterministic feedback control}

Here we consider the (deterministic) continuous-feedback Schrödinger equation

$$
i \hbar \frac{\partial \psi(t)}{\partial t}=\left[H_{0}+\mathrm{C}\left(\left\langle\psi(t)\left|O_{C}\right| \psi(t)\right\rangle\right)\right] \psi(t)
$$

which conceptually follows from a sequence of laboratory experiments measuring some observable $O_{c}$ at an increasing set of measurement times, in the limit that the intervals between these times are vanishingly small. In this approach, the probabilistic effect introduced by a measurement at each subsequent time is avoided by 'discarding' the quantum system after each measurement, using the measurement information to extend the control law over the following interval, and repeating the experiment up until the next measurement time.

Different choices of $C(\cdot)$ and $O_{C}$ may result in Eqn. (21.1) having qualitatively diverse behavior. An interesting case exists (Demiralp and Rabitz (1997)) under the assumptions (i) that $O_{C}=\delta\left(\mathbf{x}-\mathbf{x}^{\prime}\right)$ is the Dirac delta operator, and (ii) that the control law is $C\left(|\psi(\mathbf{x}, t)|^{2}\right)=-\gamma|\psi(\mathbf{x}, t)|^{2}$, where $\gamma$ is a positive constant. The resulting equation

$$
i \hbar \frac{\partial \psi(\mathbf{x}, t)}{\partial t}=-\left[\frac{\hbar^{2}}{2 m} \nabla^{2}+\gamma|\psi(\mathbf{x}, t)|^{2}\right] \psi(\mathbf{x}, t)
$$

admits dispersion free solutions (i.e., it preserves $\left.|\psi(\mathbf{x}-\mathbf{v} t)|^{2}\right)$ and also solitonic solutions under suitable conditions, cf. Lamb Jr. (1980); Drazin and Johnson (1989). These types of stable solutions may be significant in many applications of quantum control, including quantum information theory. Equation (21.2) may also be derived from the quantum optimal control formalism under the assumptions stated above; thus, dispersion free control solutions are optimal under these same conditions. The existence of such a control law invites a search for other general classes of control Hamiltonians $H_{0}+C\left(\left\langle O_{c}(t)\right\rangle\right)$ that exhibit non-dispersive or other distinct types of qualitative behavior of practical interest. 


\section{Measurement disturbances and feedback control}

This section is concerned with the effects of taking real-time measurements on a single quantum system while it is being controlled (here, "single" implies that the sequential "measure and discard" approach of the previous section is abandoned). Feedback may augment learning or optimal control methods by providing real-time information about the evolving quantum system for the stabilization of particularly sensitive objectives (e.g., locking a quantum system around an unstable point on its potential energy surface). This scenario naturally arises in the implementation of a feedback control law where measurements are taken at a discrete set of times $\left\{t_{i}\right\}$ : the control law may be written as

$$
C(t)=C\left(\left\langle\psi\left(t_{i}\right)\left|O_{c}\right| \psi\left(t_{i}\right)\right\rangle\right) \quad, \quad t_{i} \leq t
$$

There exist well-established procedures for determining feedback control laws based on measurements of evolving deterministic and stochastic classical systems in engineering control (e.g., Sontag (1998); Sotine and Li (1991)), and it is possible that many of these methods may be adapted to quantum mechanical control problems. Extensive consideration has been given to the effects of measurements on evolving quantum mechanical systems, including analysis in the contexts of continuous feedback and the control of quantum systems by homodyne detection (i.e., measurement of a component of the light field) cf. Carmichael (1999a,b); Wiseman and Milburn (1993b,a); Braginsky and Khalili (1992); Wiseman (1994, 1993); Hofmann et al. (1998b,a); Carmichael (1996). These works generally treat the more difficult problem of random measurement times; here, we give only an elementary discussion of ideas relevant to feedback control with measurements taken at a deterministic, discrete set of times.

A postulate of quantum mechanics states that a perfectly precise measurement of an operator $O_{c}$ (with nondegenerate spectrum) on a finite-dimensional Hilbert space must both yield one of the eigenvalues $\lambda_{j}$ of the operator and result in a disturbance such that $\psi(t)$ collapses to the associated eigenstate $\psi_{j}$. The measurement process introduces a stochastic element into the evolution of the quantum system, with the probability of collapse into $\psi_{j}$ being $\left|\left\langle\psi_{j} \mid \psi(t)\right\rangle\right|^{2}$. Each measurement in feedback quantum control therefore involves an information tradeoff: the system is perturbed away from the deterministic Schrödinger equation, but a measurement is used to update the control law. These random transitions, and the evolution they determine on intervals between the $t_{i}$ via the control laws (21.3), determine a "stochastic quantum map" (e.g. Carmichael (1999b)) between states at these times. If the measurement process does not completely determine the quantum states (or if mixed states are present for other reasons), a formulation involving a stochastic map between conditioned density operators is required, cf. Carmichael (1999b). 


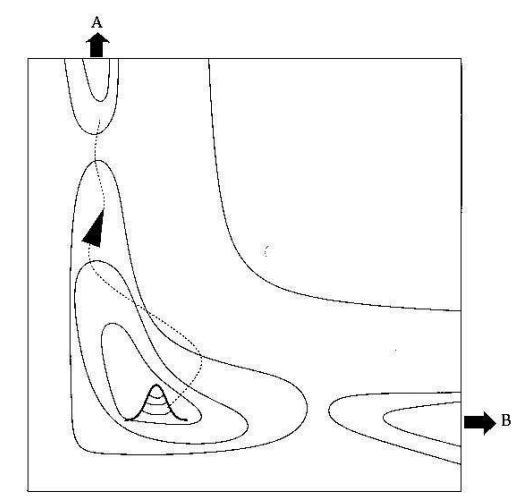

Fig. 6. Schematic illustration of a wave packet track evolving on a potential surface (contours shown). For control a goal may be to find the field $\epsilon(t)$ that will steer the track out of product channel A or B, while for inversion the track is observed in the laboratory and the goal is to determine the potential in the regions traveled by the track, cf. Lu and Rabitz (1995).

A crucial unresolved question is what general classes of quantum problems will be assisted by incorporating feedback measurements. More specifically, it may be possible to show that taking a certain number of measurements improves control (with reasonable assumptions about the problem-dependent frequency and timing of measurements to optimize the feedback quantum control problem). A related matter is the possibility of making "weak observations" that give useful information about a quantum system while introducing only minimal perturbations. The effects of measurements on the feedback control process for systems satisfactorily described semiclassically also remain to be characterized, and this domain may be especially amenable to performing weak measurements.

\section{Closing the loop through machine feedback}

The final topic on feedback control is the possibility of closing the control loop in laboratory hardware through machine feedback. Recent work in acoustics illustrates the capability of focusing reflected waves back upon their sources cf. Fink (1999); Fink and Prada (1996) in an iterative fashion in order to enhance the intensity in the focal volume. An analogy of this technique relevant to quantum mechanics might be "reflection" through special measurement devices that could then send modified electromagnetic waves precisely back to an emitting quantum mechanical source to better achieve the control objectives. This process may be fully quantum mechanical if carried out in a suitable optical cavity, but in general the same closed loop observation/disturbance issues raised in the previous section must be considered here. At this juncture such a machine is only a gedanken process, but its potential strongly motivates an analysis of the concept. 


\section{Algorithms for the inversion of quantum dynamics data}

Knowledge of the potential $V$ and the dipole $\mu$ (or other coupling coefficients) is required for control law design and is of fundamental importance to many other applications in chemistry and physics. This section concerns dynamical algorithms that invert time-dependent laboratory data to identify these operators. This problem of determining $\mu(\mathbf{x})$ or $V(\mathbf{x})$ may be related (Lu and Rabitz (1995); see also Bargheer et al. (1999) for a different approach) to the problem of determining the control law $C(t)$ (of the form (6.1)) that will cause a quantum system to follow a prescribed track (see Figure 6). In particular, if the expectation values $y(t) \equiv\left\langle O_{h}(t)\right\rangle$ of a time-independent operator $O_{h}$ are established from a series of observations of an evolving quantum system, the Schrödinger equation and the Heisenberg equation of motion form the pair of coupled (forward-inverse) equations

$$
\begin{aligned}
& i \hbar \frac{d \psi(t)}{d t}=\left[H_{0}-\mu \epsilon(t)\right] \psi(t) \\
& i \hbar \frac{d y(t)}{d t}=\left\langle\psi(t)\left|\left[H_{0}-\mu \epsilon(t), O_{h}\right]\right| \psi(t)\right\rangle .
\end{aligned}
$$

The solution of these evolution equations may in principle be attempted for any two unknowns. As knowledge of $\psi(t)$ is not available in any physical problem, the wave function will always be considered as one of these unknowns; the other may be chosen from either $\epsilon(t), \mu(\mathbf{x})$, or $V(\mathbf{x})$ with the complementary pair assumed as known. The first of these possibilities was treated in Section 16, where the fact that the expectation value on the right hand side of Eqn. (22.2) involves only spatial integration was exploited to write $\epsilon(t)$ explicitly in the form of Eqn. (16.3). We now turn to the solution for $\mu(\mathbf{x})$ and $V(\mathbf{x})$.

Equation (22.2) may be rewritten (cf. Lu and Rabitz (1995)) as a Fredholm integral equation of the second type, after regularization enforcing the physical criterion that $\mu(\mathbf{x})$ and $V(\mathbf{x})$ should decay as $x \rightarrow \infty$. The structure of this equation is

$$
\int_{-\infty}^{\infty} \kappa\left(x, x^{\prime}\right) f\left(x^{\prime}\right) d x^{\prime}+\alpha f(x)=h(x),
$$

where $f(x)$ is the unknown ( $\mu$ or $V), \alpha$ is the regularization parameter, and the kernel $\kappa\left(x, x^{\prime}\right)$ and the inhomogeneity $h(x)$ involve the data $y(t)$, the operator $O_{h}$, and the solution $\psi(t)$ to the Schrödinger equation (22.1). Solution of the regularized pair of Eqns. (22.1) and (22.3) may be accomplished using the tracking procedure discussed in Section 16, with the role of $\epsilon(t)$ replaced by $\mu(\mathbf{x})$ or $V(\mathbf{x})$. The procedure consists of formally solving Eqn. (22.3) for $f(x)$ and substituting the result into the Schrödinger equation (22.1) to solve for 
$\psi(t)$, which is then used to determine $f(x)$ in a final inversion step.

In the special case that the data being inverted is the probability density function $|\psi(\mathbf{x}, t)|^{2}$ (formally, the expectation value of the Dirac delta operators $\delta\left(\mathbf{x}-\mathbf{x}^{\prime}\right)$ ) (see Krause et al. (1997)), a direct algorithm has been developed to identify $V(\mathbf{x})$ without the expensive requirement of numerically solving the Schrödinger equation cf. Zhu and Rabitz (1999b). The algorithm relies on Ehrenfest's relation, and appears to be difficult to generalize to other measurement operators.

In general, the goal is to solve Eqns. (22.1) and (22.2) with minimum of distortion introduced by additional criteria; in the example above, the balance between this objective and stability requirements is set by $\alpha$, whose optimal value depends on the details of the particular problem and solution method. Additionally, the evolution of the quantum system which determines $\kappa\left(x, x^{\prime}\right)$ in (22.3) is in turn governed by the applied field $\epsilon(t)$ in Eqn. (22.1). Hence, it should be possible to determine a control law which allows inversion with maximum stability to produce optimal dynamical regularization. Note that meaningful inversion of Eqns. (22.1) and (22.2) may only be expected if the control law $\epsilon(t)$ steers the wave function to be non-zero in the domain in which $\mu$ or $V$ is to be determined; the formulation of Eqns. (22.1) and (22.2) may be extended to incorporate multiple realizations of the control law $\epsilon_{j}(t)$ (see Lu and Rabitz (1995)) that, taken together, may provide the desired evolution over the entire spatial domain of interest. However, for dynamical reasons the kernel $\kappa\left(x, x^{\prime}\right)$ may still produce a singular operator in Eqn. (22.3) where it is significantly non-zero. The additional conditions required to resolve this problem are not immediately apparent.

A complete laboratory device may be envisioned to function as an optimal dynamics inversion machine for the efficient and automatic discovery of $\mu$ or $V$ for diverse quantum systems (cf. Rabitz and Shi (1991); Rabitz and Zhu (2000)). This machine would operate in a closed-loop mode to take advantage of the ability to perform a very large number of high throughput control-observation experiments, and would operate through the following steps, sketched in Fig. 7: (1) Initial approximations for $V$ and $\mu$ could be used to design an optimal control field aimed at causing the wave packet to evolve in desired spatial areas where $V$ and $\mu$ are being sought. (2) Laboratory experiments using this control law would be performed to produce the data trajectory $\left\langle O_{h}(t)\right\rangle=y(t)$. (3) An inversion would be performed to produce updated potential or dipole information. (4) If the spatial domain of interest was not completely covered by the current trajectory or if the inversion quality is not adequate, the procedure would be repeated with the assistance of partial Hamiltonian information gained from step 3 . 


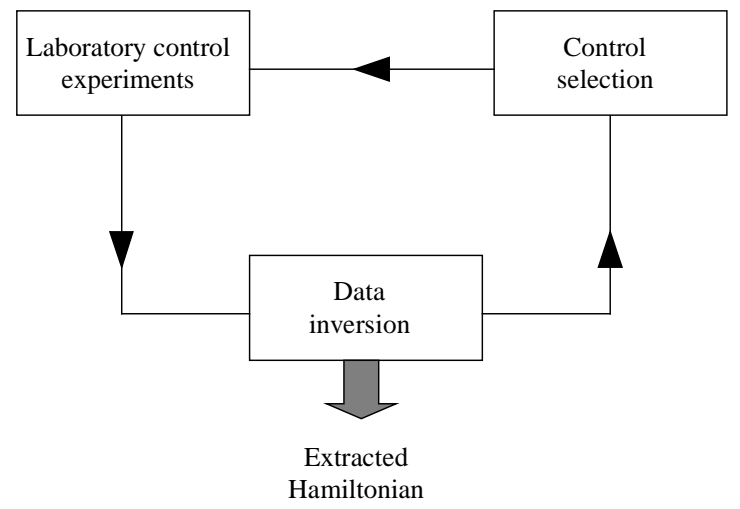

Fig. 7. The optimal dynamics identification machine.

\section{Identification of quantum control mechanism and "rules of thumb"}

A cornerstone of chemistry is that physically similar molecules tend to exhibit similar chemical behavior. The emphasis is on "similar" and in the context of quantum control the criteria for defining similarity is not known. From the rich behavior and information content in the design, closed loop, and dynamical inversion aspects of quantum control, one can anticipate using the emerging results to provide insight or estimates for the control laws for physically related, but as yet uninvestigated, problems. The body of relationships (as just yet beginning to be observed) between quantum systems, control objectives, and control laws may be called quantum control rules of thumb. A specialized example is the explanation of the timing of the pulses used in the STIRAP control method (cf. Rice and Zhao (2000)). However, attempts to find general rules have proved much more difficult than was at first expected. Nevertheless, the implications of these rules for both the theory of quantum control and its practical implementation are substantial: resolution of this matter may be the most important challenge ahead for the field.

A natural strategy for identifying rules of thumb might ensue from a type of quantum mechanical reverse engineering: solutions $\{C(t), \psi(t), \lambda(t),\langle O\rangle\}$ to the optimal control equations, or $C(t)$ and $\langle O\rangle$ from closed loop experiments, could provide a physical basis for understanding the mechanisms and pathways leading the quantum system from initial conditions to final control objectives. However, there exist many examples in the literature in which the structure of the final control fields and the resulting control pathways are found to be highly nonintuitive, and judging the relevance of such solutions in terms of general rules of thumb is difficult in the presence of a possibly large number of (locally) optimal solutions. Further insight into the structure of these local minima may be gained by identifying the family of locally optimal control solutions and enumerating them based on their optimality. This problem might be partially alleviated by incorporating a global search procedure in the op- 
timization algorithm (e.g., a genetic algorithm), both for theoretical design and laboratory control. If such techniques were developed, the existence of multiple solutions could possibly be exploited as a large body of data about control behavior.

In the context of closed-loop laboratory implementation of controls, the presence of multiple solutions to the quantum optimal control problem opens up several options (Geremia et al. (2000)). Given that there exist many possible solutions $C(t)$ from which identification of control mechanisms could be attempted, it is important to select solutions that contain a minimum of extraneous information that detract from this task. In addition, control rules of thumb would best be developed based on solutions that are robust to realistic laboratory noise. Both the suppression of extraneous structural components in $C(t)$ and the selection of robust control fields may be accomplished through the use of appropriate cost functionals (Geremia et al. (2000)). This "cleanup" of control laws is likely to assist in identifying rules of thumb for the control of quantum systems.

A first step toward identifying quantum control rules of thumb involves the effective classification of similarities and differences between molecules in a context relevant to the controls directing them to certain physical objectives. This type of classification is fundamental in many fields of chemistry and physics, in which the vast numbers of molecules are categorized according to their relevant behaviors or properties. However, presently the standard measures have not been able to consistently predict the structure of control fields for particular objectives.

A three-way classification structure will be necessary, relating (i) control laws, (ii) molecular Hamiltonians and coupling terms, and (iii) and control objectives. Progress toward (i) will likely involve identification of the relevant properties of control laws $C(t)$. Experience thus far suggests that the most useful features will include, but extend beyond, description of spectral components and intensities. Progress may also follow from exploring the relation between the form of the Hamiltonian and (sets of) control solutions for a fixed particular control objective, possibly through numerical optimal control calculations for a series of quantum systems whose Hamiltonians differ by small increments, but collectively cover a broad sampling of physical systems.

\section{Chapter 6. Conclusion}

This article aimed to present an overview of the current state of attempts to control quantum phenomena. Special emphasis was given to the conceptual, algorithmic and numerical aspects of the subject. In envisioning further advances along these lines it is very important to explicitly consider the special capabilities of performing massive numbers of control experiments over a short 
laboratory time. In many respects the field of control over quantum phenomena is a field which is young with the bulk of its developments lying ahead. It is hoped that this article provides some stimulus to push the field further.

Acknowledgements: H.R and E.B. acknowledge support from the National Science Foundation and the Department of Defense. E. B. was supported by a NSF Graduate Fellowship.

G.T. would like to thank Wusheng Zhu from the Princeton University for helpful discussions and remarks.

\section{References}

Ackerman, J. (Ed.), 1985. Uncertainty and Control, Proceedings of an International Seminar, Bonn, Germany, May 1985. Springer.

Agarwal, G., 1997. Nature of the quantum interference in electromagneticfield-induced control of absorption. Phys. Rev. A 55, 2467-2470.

Albertini, F., D'Alessandro, D., 2001. Notions of controllability for quantum mechanical systems. Preprint arXiv:quant-ph/0106128 .

Assion, A., Baumert, T., Bergt, M., Brixner, T., Kiefer, B., Seyfried, V., Strehle, M., Gerber, G., 1998. Control of chemical reactions by feedbackoptimized phase-shaped femtosecond laser pulses. Science 282, 919-922.

Athans, M., Falb, P. L., 1966. Optimal Control. McGraw-Hill.

Bacon, D., Lidar, D. A., Whaley, K. B., 1999. Robustness of decohrence-free subspaces for quantum computation. Phys. Rev. A 60, 1944-1955.

Ball, J., Marsden, J., Slemrod, M., 1982. Controllability for distributed bilinear systems. SIAM J.Control and Optimization 20 (4).

Bardeen, C., Yakovlev, V. V., Wilson, K. R., Carpenter, S. D., Weber, P. M., Warren, W. S., 1997a. Feedback quantum control of molecular electronic population transfer. Chem. Phys. Lett. 280, 151-158.

Bardeen, C. J., Cao, J., Brown, F. L. H., Wilson, K. R., 1999. Using timedependent rate equations to describe chirped pulse excitation in condensed phases. Chem. Phys. Lett. 302, 405-410.

Bardeen, C. J., Che, J., Wilson, K. R., Yakovlev, V. V., Apkarian, V. A., Martens, C. C., Zadoyan, R., Kohler, B., Messina, M., 1997b. Quantum control of $I_{2}$ in the gas phase and in condensed phase solid kr matrix. J. Chem. Phys. 106, 8486-8503.

Bardeen, C. J., Che, J., Wilson, K. R., Yakovlev, V. V., Cong, P., Kohler, B., Krause, J. L., Messina, M., 1997c. Quantum control of NaI photodissociation reaction product states by ultrafast tailored light pulses. J. Phys. Chem. A 101, 3815-3822.

Bardeen, C. J., Yakovlev, V. V., Squier, J. A., Wilson, K. R., 1998. Quantum control of population transfer in green flourescent protein by using chirped femtosecond pulses. J. Am. Chem. Soc. 120, 13023-13027. 
Bargheer, M., Dietrich, P., Donovang, K., Schwentner, N., 1999. Extraction of potentials and dynamics from condensed phase pump-probe spectra: Application to $I_{2}$ in Kr matrices. J. Chem. Phys. 111, 8556-8564.

Bartana, A., Kosloff, R., 1993. Laser cooling of molecular internal degrees of freedom by a series of shaped pulses. J. Chem. Phys. 99, 196-210.

Bartana, A., Kosloff, R., 1997. Laser cooling and internal degrees of freedom. II. J. Chem. Phys. 106, 1435-1448.

Bartels, R., Backus, S., Zeek, E., Misoguti, L., Vdovin, G., Christov, I. P., Murnane, M. M., Kapteyn, H. C., 2000. Shaped-pulse optimization of coherent emission of high-harmonic soft x-rays. Nature 406, 164-166.

Bergt, M., Brixner, T., Kiefer, B., Strehle, M., Gerber, G., 1999. Controlling the femtochemistry of $\mathrm{Fe}(\mathrm{CO}) 5$. J. Phys. Chem. A. 103, 10381-10387.

Beumee, J., Schwieters, C., Rabitz, H., 1990. Optical control of molecular motion with robustness and application to vinylidene fluoride. J. Opt. Soc. America B , 1736-1747.

Beumee, J. G. B., Rabitz, H., 1992. Robust optimal control theory for selective vibrational excitation in molecules: A worst case analysis. J. Chem. Phys. 97, 1353-1364.

Braginsky, V., Khalili, F., 1992. Quantum Measurement. Cambridge University Press.

Brockett, R., 1970. Finite Dimensional Linear Systems. Wiley.

Brown, E., Rabitz, H., in press. Some mathematical and algorithmic challenges in the control of quantum dynamics phenomena. J. Math. Chem. .

Brown, F., Sibley., R., 1998. Quantum control for arbitrary linear and quadratic potentials. Chem. Phys. Lett. 292, 357-368.

Cancès, E., Le Bris, C., 1999. On the time-dependent Hartree-Fock equations coupled with a classical nuclear dynamics. Mathematical Models and Methods in Applied Sciences 9 (7), 963-990.

Cancès, E., Le Bris, C., Mathieu, P., 2000. Bilinear optimal control of a Schrödinger equation. C. R. Acad. Sci. Paris Sér. I Math. 7, 567-571.

Cao, Messina, M., Wilson, K., 1997. Quantum control of dissipative systems: exact solutions. J. Chem. Phys. 106, 5239-5248.

Cao, J., Wilson., K., 1997. A simple physical picture for the quantum control of wave packet localization. J. Chem. Phys. 107, 1441-1450.

Carmichael, H., 1996. Stochastic Schrodinger equations: What they mean and what they can do. In: Eberly, J., Mandel, L., Wolf, E. (Eds.), Coherence and Quantum Optics VII,. p. 177.

Carmichael, H., 1999a. Master Equations and Fokker Plank Equations. Springer.

Carmichael, H., 1999b. An Open Systems Approach to Quantum Optics. Springer.

Chen, Y., Gross, P., Ramakrishna, V., Rabitz, H., Mease, K., 1995. Competitive tracking of molecular objectives described by quantum mechanics. J. Chem. Phys. 102, 8001-8010.

Christofides, N., 1975. Graph Theory: An Algorithmic Approach. Academic 
Press.

Chuang, I. L., Laflamme, R., Shor, P. W., Zurek, W. H., 1995. Quantum computers, factoring, and decoherence. Science 270, 1633-1635.

Cohen-Tannoudji, C., Liu, B., Laloë, F., 1997. Quantum Mechanics. Wiley.

Combariza, J., Just, B., Manz, J., Paramonov, G., 1991. Isomerization Controlled by Ultrashort Infrared Laser Pulses: Model Simulations for the Inversion of Ligands $(\mathrm{H})$ in the Double-Well potential of an Organometallic Compound. [(C5H5)(CO)2FePH2]. J Phys Chem 95, 10351.

Dardi, P. S., Gray, S. K., 1982. Classical and quantum-mechanical studies of hf in an intense laser field. J. Chem. Phys. 77, 1345-1353.

Davis, M. J., Heller, E. J., 1984. Comparisons of classical and quantum dynamics for initially localized states. J. Chem. Phys. 80, 5036-5048., this and the following three references are from the list given in note 18 of Schwieters and Rabitz (1993).

de Araujo, L., Walmsley, I., 1999. Quantum control of molecuar wavepackets: an approximate analytic solution for the strong-response regime. J. Phys. Chem. A 103, 10409-10416.

Demiralp, M., Rabitz, H., 1993. Optimally controlled quantum molecular dynamics: A perturbation formulation and the existence of multiple solutions. Phys. Rev. A 47, 809-816.

Demiralp, M., Rabitz, H., 1997. Dispersion-free wavepackets and feedback solitonic motion in controlled quantum dynamics. Phys. Rev. A. 55, 673677.

Demiralp, M., Rabitz, H., 1998. Assessing optimality and robustness of control over quantum dynamics. Phys. Rev. A 57, 2420-2425.

Dion, C., Bandrauk, A., Atabek, O., Keller, A., Umeda, H., Fujimura, Y., 1999a. Two-frequency IR laser orientation of polar molecules. numerical simulations for HCN. Chem. Phys.Lett 302, 215-223.

Dion, C., Keller, A., Atabek, O., Bandrauk, A., 1999b. Laser-induced alignment dynamics of HCN: Roles of the permanent dipole moment and the polarizability. Phys. Rev. A 59(2), 1382.

Dorato, P. (Ed.), 1987. Robust Control. IEEE Selected Reprint Series. IEEE Press, new York.

Drazin, P., Johnson, R., 1989. Solitions: an Introduction. Cambridge University Press.

Duan, L., Guo, G., 1999. Suppressing environmental noise in quantum computation through pulse control. Phys. Lett. A 261, 139-144.

Fink, M., 1999. Time reversed acoustics. Sci. Am. , 91-97.

Fink, M., Prada, C., 1996. Ultrasonic focusing with time-reversal mirrors. In: Briggs, A., Arnold, W. (Eds.), Advances in Acoustic Microscopy Series. Vol. 2. pp. 219-251.

Fu, H., Schirmer, S., Solomon, A., 2001. Complete controllability of finite-level quantum systems. J. Phys. A. 34.

Gammaitoni, L., 1998. Stochastic resonance. Rev. Mod. Phys. 70, 223-287.

Geremia, J., Weiss, E., Rabitz, H., 2001. Achieving the laboratory control 
of quantum dynamics phenomena using nonlinear functional maps. Chem. Phys. 267, 209-222.

Geremia, J., Zhu, W., Rabitz, H., 2000. Incorporating physical implementation concerns into closed loop quantum control experiments. J. Chem. Phys. 113, 10841-10848.

Girardeau, M. D., Ina, M., Schirmer, S. G., Gulsrud, T., 1997. Kinematical bounds on evolution and optimization of mixed quantum states. Phys. Rev. A 55, R1565-R1568.

Girardeau, M. D., Schirmer, S. G., Leahy, J. V., Koch, R. M., 1998. Kinematical bounds on optimization of observables for quantum systems. Phys. Rev. A 58, 2684-2689.

Goldberg, D., 1989. Genetic Algorithms in Search, Optimization, and Machine Learning. Addison-Wesley.

Gross, P., Neuhauser, D., Rabitz, H., 1993a. Teaching lasers to control molecules in the presence of laboratory field uncertainty and measurement imprecision. J. Chem. Phys. 98, 4557-4566.

Gross, P., Singh, J., Rabitz, H., Huang, G., Mease, K., 1993b. Inverse quantum-mechanical control: A means for design and a test of intuition. Phys. Rev. A 47, 4593-4603.

Gronager, M., Henriksen., N., 1998. Real-time control of electronic motion: Application to NaI. J. Chem. Phys. 109, 4335-4341.

Harel, G., Akulin, V. M., 1999. Complete control of Hamiltonian quantum systems: Engineering of floquet evolution. Phys. Rev. Lett. 82, 1-5.

Hofmann, H., Hess, O., Mahler, G., 1998a. Quantum control by compensation of quantum fluctuations. Optics Express 2, 339-346.

Hofmann, H. F., Mahler, G., Hess, O., 1998b. Quantum control of atomic systems by homodyne detection and feedback. Phys. Rev. A. 57, 4877-4888.

Hoki, K., Ohtsuki, Y., Kono, H., Fujimura, Y., 1999. Quantum control of NaI predissociation in subpicosecond and several-picosecond time regimes. J. Phys. Chem. A 103, 6301-6308.

Hornung, T., Meier, R., Motzkus, M., 2000. Optimal control of molecular states in a learning loop with a parameterization in frequency and time domain. Chem. Phys. Lett. 326, 445-453.

Hosoe, S. (Ed.), 1991. Robust Control, Proceedings of a Workshop held in Tokyo, Japan, June 23-24, 1991. Springer.

Huang, G., Tarn, T., Clark, J., 1983. On the controllability of quantummechanical systems. J. Math. Phys. 24.

Judson, R., Lehmann, K., Rabitz, H., Warren, W. S., 1990. Optimal design of external fields for controlling molecular motion - application to rotation. J. Molec. Structure 223, 425-456.

Judson, R., Rabitz, H., 1992. Teaching lasers to control molecules. Phys. Rev. Lett .

Kailath, T., 1980. Linear Systems. Prentice Hall.

Khalil, H. K., 1996. Nonlinear systems. Macmillan, New York.

Kobayashi, M., Nov. 1998. Mathematics makes molecules dance. Siam News 
$31(9), 24$.

Krause, J., Schafer., K., 1999. Control of thz emission from stark wave packets. J. Phys. Chem. A 103, 10118-10125.

Krause, J. L., Reitze, D. H., Sanders, G. D., Kuznetsov, A. V., Stanton, C. J., 1998. Quantum control in quantum wells. Phys. Rev. B 57, 9024-9034.

Krause, J. L., Schafer, K. J., Ben-Nun, M., Wilson, K. R., 1997. Creating and detecting shaped Rydberg wave packets. Phys. Rev. Lett. 79, 4978-4981.

Krotov, V., 1973. Optimization methods of control with minimax criteria. i. Automat. Remote Control 34, 1863-1873, translated from Avtomat. i Telemeh. 1973, , no. 12, 5-17.

Krotov, V., 1974a. Optimization methods in control processes with minimax criteria. ii. Automat. Remote Control 35, translated from Avtomat. i Telemeh. 1974, , no. 1, 5-15.

Krotov, V., 1974b. Optimization methods of control with minimax criteria. iii. Automat. Remote Control 35, 345-353, translated from Avtomat. i Telemeh. 1974 , no. 3, 5-14.

Kulander, K. C., Orel, A. E., 1981. Laser-collision induced chemical-reactions - a comparison of quantum-mechanical and classical-model results. J. Chem. Phys. 75, 675-680.

Kunde, J., Baumann, B., Arlt, S., Morier-Genoud, F., Siegner, U., Keller, U., 2000. Adaptive feedback control of ultrafast semiconductor nonlinearities. Appl. Phys. Lett. 77, 924.

Lamb Jr., G., 1980. Elements of Soliton Theory. Wiley.

Leforestier, C., 1986. Competition between dissociation and exchange processes in a collinear A + BC collision - comparison of quantum and classical results. Chem. Phys. Lett. 125, 373-377.

Levis, R. J., Menkir, G., Rabitz, H., 2001. Selective bond dissociation and rearrangement with optimally tailored, strong-field laser pulses. Science 292, 709-713.

Li, B., Turinici, G., Ramakhrishna, V., Rabitz, H., 2002. Optimal dynamic discrimination of similar molecules through quantum learning control. submitted to J. Phys. Chem. A .

Lidar, D., Chuang, I. L., Whaley, K. B., 1998. Decohrence-free subspaces for quantum computation. Phys. Rev. Lett. 81, 2594-2597.

Lidar, D. A., Bacon, D., Whaley, K. B., 1999. Concatenating decoherencefree subspaces with quantum error correcting codes. Phys. Rev. Lett. 82, 4556-4559.

Lu, Z. M., Rabitz, H., 1995. Unified formulation for control and inversion of molecular dynamics. J. Phys. Chem. 99, 13731-13735.

Luenberger, D., 1979. Introduction to Dynamic Systems: Theory, Models, and Applications. Wiley.

Meshulach, D., Silberberg., Y., 1998. Coherent quantum control of two-photon transitions by a femtosecond laser pulse. Nature 396, 239-241.

Mishima, K., Yamashita., K., 1999a. Quantum control of photodissociation wavepackets. J. Molec. Structure 461-462, 483-491. 
Mishima, K., Yamashita., K., 1999b. Theoretical study on quantum control of photodissociation and photodesorption dynamics by femtosecond chirped laser pulses. J. Chem. Phys. 110, 7756-7769.

Mohler, R., 1983. Bilinear Control Processes. Academic Press.

Nijmeiher, H., van der Schaft, A., 1990. Nonlinear Dynamical Control Systems. Springer.

Ohtsuki, Y., Kono, H., Fujimura, Y., 1998. Quantum control of nuclear wave packets by locally designed optimal pulses. J. Chem. Phys. 109, 9318-9331.

Ohtsuki, Y., Zhu, W., Rabitz, H., 1999. Monotonically convergent algorithm for quantum optimal control with dissipation. J. Chem. Phys. 110, 98259832.

Ong, C. K., Huang, G. M., Tarn, T. J., Clark, J. W., 1984. Invertability of quantum-mechanical control systems. Math. Systems Theory 17, 335-350.

Pastirk, I., Brown, E. J., Zhang, Q., Dantus, M., 1998. Quantum control of the yield of a chemical reaction. J. Chem. Phys. 108, 4375-4378.

Peirce, A., Dahleh, M., Rabitz, H., 1988. Optimal control of quantum mechanical systems: Existence, numerical approximations, and applications. Phys. Rev. A 37, 4950-4964.

Phan, M. Q., Rabitz, H., 1997. Learning control of quantum-mechanical systems by laboratory identification of effective input-output maps. Chem. Phys. 217, 389-400.

Phan, M. Q., Rabitz, H., 1999. A self-guided algorithm for learning control of quantum-mechanical systems. J. Chem. Phys. 110, 34-41.

Rabitz, H., de Vivie-Riedle, R., Motzkus, M., Kompa, K., 2000. Wither the future of controlling quantum phenomena? Science 288, 824-828.

Rabitz, H., Shi, S., 1991. Optimal control of molecular motion: Making molecules dance. In: Bowman., J. (Ed.), Advances in Molecular Vibrations and Collision Dynamics. Vol. 1, Part A. JAI Press, Inc., pp. $187-214$.

Rabitz, H., Zhu, W., 2000. Optimal control of molecular motion: Design, implementation, and inversion. Acc. Chem. Res. 33, 572-578.

Ramakrishna, V., Nov. 2000. Private communication.

Ramakrishna, V., Salapaka, M., Dahleh, M., Rabitz, H., Pierce, A., 1995. Controllability of molecular systems. Phys. Rev. A 51 (2), 960-966.

Ramakrishna et. al., V., 2000. Explicit generation of unitary transformations in a single atom or molecule. Phys. Rev. A 61, 032106-1 - 032106-6.

Rice, S., Zhao, M., 2000. Optical Control of Quantum Dynamics. Wiley, many additional references to the subjects of this paper may also be found here.

Schirmer, S., Girardeau, M., Leahy, J., 2000. Efficient algorithm for optimal control of mixed-state quantum systems. Phys. Rev. A 61, 012101.

Schirmer, S. G., Fu, H., Solomon, A., 2001. Complete controllability of quantum systems. Phys. Rev. A 63, 063410.

Schwieters, C., Rabitz, H., 1993. Optimal control of classical systems with explicit quantum/classical difference reduction. Phys. Rev. A 48, 2549-2457.

Schwieters, C., Rabitz, H., unpublished results. Designing time-independent classically equivalent potentials to reduce quantum/classical observable dif- 
ferences using optimal control theory.

Sepulchre, R., Jankovic, M., Kokotovic, P., 1998. Constructive Nonlinear Control. Springer.

Shah, S., Rice, S., 1999. Controlling quantum wavepacket motion in reduceddimensional spaces: reaction path analysis in optimal control of hen isomerization. Faraday Discuss. 113, 319-331.

Shapiro, M., Brumer, P., 1989. Coherent chemistry: Controlling chemical reactions with lasers. Acc. Chem. Res. 22, 407.

Shen, H., Dussault, J.-P., Bandrauk, A., 1994. Optimal pulse shaping for coherent control by the penalty algorithm. Chem. Phys. Lett. 221, 498-506.

Shen, L., Shi, S., Rabitz, H., Lin, C., Littman, M., Heritage, J. P., Weiner, A. M., 1993. Optimal control of the electric susceptibility of a molecular gas by designed non-resonant laser pulses of limited amplitude. J. Chem. Phys. 98, 7792-7803.

Shi, S., Woody, A., Rabitz, H., 1988. Optimal control of selective vibrational excitation in harmonic linear chain molecules. J. Chem. Phys. 88, 68706883.

Shor, P., 1995. Scheme for reducing decoherence in quantum computer memory. Phys. Rev. A. 52, R2493-R2496.

Somlói, J., Kazakov, V., Tannor, D., 1993. Controlled dissociation of $i_{2}$ via optical transitions between the X and B electronic states. Chem. Phys. 172, 85-98.

Sontag, 1998. Mathematical Control Theory. Springer, and references within. Sotine, J. J., Li, W., 1991. Applied Nonlinear Control. Prentice Hall.

Steane, M., 1998. Introduction to quantum error correction. Phil. Trans. Roy. Soc. London Ser A 356, 1739-1758.

Tannor, D., Bartana, A., 1999. On the interplay of control fields and spontaneous emission in laser cooling. J. Chem. Phys. A. 103, 10359-10363.

Tannor, D., Kazakov, V., Orlov, V., 1992. Control of photochemical branching: Novel procedures for finding optimal pulses and global upper bounds. In: Broeckhove J., Lathouwers L. (Eds.), Time Dependent Quantum Molecular Dynamics. Plenum, pp. 347-360.

Tannor, D., Rice, S., 1985. Control of selectivity of chemical reaction via control of wave packet evolution. J. Chem. Phys 83, 5013-5018.

Tannor, D. J., Kosloff, R., Bartana, A., 1999. Laser cooling of internal degrees of freedom of molecules by dynamically trapped states. Faraday Discuss. $113,365-383$.

Tersigni, S. H., Gaspard, P., Rice, S., 1990. On using shaped light-pulses to control the selectivity of product formation in a chemical-reaction - an application to a multiple level system. J. Chem. Phys. 93, 1670-1680.

Tóth, G., Lörincz, A., Rabitz, H., 1994. The effect of control field and measurement imprecision on laboratory feedback control of quantum systems. J. Chem. Phys. 101, 3715-3722.

Turinici, G., 2000a. Analysis of numerical methods of simulation and control in Quantum Chemistry. Ph.D. thesis, University of Paris VI, Paris, France. 
Turinici, G., December 2000b. Controllable quantities for bilinear quantum systems. In: Proceedings of the 39th IEEE Conference on Decision and Control, Sydney, Australia,. Vol. 2. pp. 1364-1369.

Turinici, G., 2000c. On the controllability of bilinear quantum systems. In: Defranceschi, M., Le Bris, C. (Eds.), Mathematical models and methods for ab initio Quantum Chemistry. Vol. 74 of Lecture Notes in Chemistry. Springer, pp. 75-92.

Turinici, G., Rabitz, H., 2001a. Quantum wavefunction control. Chem. Phys. 267, 1-9.

Turinici, G., Rabitz, H., 2001b. Wavefunction controllability in quantum systems. Submitted to: J. Phys.A. .

Turinici, G., Ramakhrishna, V., Li, B., Rabitz, H., 2002. Optimal discrimination of multiple quantum systems: Controllability analysis. in preparation .

Turinici, G., Schirmer, S., 2001. On-line controllability calculator. www-rocq. inria.fr/Gabriel. Turinici/control/calculator.html.

Viola, L., Lloyd, S., 1998. Dynamical suppression of decoherence in two-state quantum systems. Phys. Rev. A 58, 2733-2744.

Vitali, D., Tombesi, P., 1999. Using parity kicks for decoherence control. Phys. Rev. A. 59, 4178-4185.

Wang, N., Rabitz, H., 1996. Optimal control of population transfer in an optically dense medium. J. Chem. Phys. , 1173-1179.

Weinacht, T., Ahn, J., Bucksbaum, P., 1999. Controlling the shape of a quantum wavefunction. Nature 397, 233-235.

Wiseman, H. M., 1993. Stochastic quantum dynamics of a continuously monitored laser. Phys. Rev. A. 47, 5180-5192.

Wiseman, M., 1994. Quantum theory of continuous feedback. Phys. Rev. A. 49, 2133-2150.

Wiseman, M., Milburn, G. J., 1993a. Quantum-theory of field-quadrature measurements. Phys. Rev. A. 47, 643-622.

Wiseman, M., Milburn, G. J., 1993b. Quantum-theory of optical feedback via homodyne detection. Phys. Rev. Lett. 70, 548-551.

Xia, H., Merriam, A., Sharpe, S., Yin, G., Harris, S. E., 1997. Electromagnetically induced transparency in atoms with hyperfine structure. Phys. Rev. A 56, R3362-R3365.

Zhang, H., Rabitz, H., 1994. Robust optimal control of quantum molecular systems in the presence of disturbances and uncertainties. Phys. Rev. A 49, $2241-2254$.

Zhao, M., Rice, S., 1994. Optimal control of product selectivity in reactions of polyatomic molecules: a reduced-space analysis. In: Hepburn, J. (Ed.), Laser techniques for State-selected and State-to-Chemistry II, ed. Vol. SPIE 2124. pp. 246-257.

Zhu, W., Botina, J., Rabitz, H., 1998. Rapidly convergent iteration methods for quantum optimal control of population. J. Chem. Phys. 108, 1953-1963. Zhu, W., Rabitz, H., 1998. A rapid monotonically convergent iteration algo- 
rithm for quantum optimal control over the expectation value of a positive definite operator. J. Chem. Phys. 109, 385-391.

Zhu, W., Rabitz, H., 1999a. Noniterative algorithms for finding quantum optimal controls. J. Chem. Phys. 110, 7142-7152.

Zhu, W., Rabitz, H., 1999b. Potential surfaces from the inversion of time dependent probability density data. J. Chem. Phys. 111, 472-480.

Zhu, W., Rabitz, H., in press. Attaining optimal controls for manipulating quantum systems. Int. J. Quant. Chem. .

Zhu, W., Smit, M., Rabitz, H., 1999. Managing dynamical singular behavior in the tracking control of quantum observables. J. Chem. Phys. 110, 19051915 . 


\section{Index}

- adjoint state, 26

- coherent molecular discrimination, 18

- connectivity graph, 16

- conservation law, 17

- cooling of quantum systems, 44

- cost functional, 24

- density-matrix-controllablity, 13

- deterministic feedback control, 45

- dynamical Lie group, 13

- electric dipole, 4

- genetic algorithm, 40

- gradient descent, 28

- input-output maps, 42

- Krotov-type algorithm, 28

- Lagrange multiplier, 26

- machine feedback, 47

- measurement disturbances and feedback control, 46

- optimal control, 6

- quantum control problem, 5

- quantum learning control, 40

- split-operator propagation scheme, 34

- stability operator, 35

- state-controllablity, 12

- tracking operator, 37

- tracking singularity, 38

- transitivity, 13

- Zhu and Rabitz's monotonically convergent algorithm, 30 